\title{
Effects of heterogeneous surface geometry on secondary flows in turbulent boundary layers
}

\author{
T. Medjnoun ${ }^{1} \dagger$, C. Vanderwel $^{1}$ and B. Ganapathisubramani ${ }^{1}$ \\ ${ }^{1}$ Aerodynamics and Flight Mechanics Research Group, University of Southampton, \\ Hampshire, SO17 1BJ, UK
}

(Received xx; revised xx; accepted $\mathrm{xx}$ )

The effect of spanwise heterogeneous surface geometry on the turbulent boundary layer secondary flows and on skin friction is investigated experimentally. The surfaces consist of smooth streamwise-aligned ridges of different shapes and widths with spanwise wavelengths comparable to the boundary layer thickness $(S / \delta \approx \mathcal{O}(1))$. Cross-stream StereoPIV combined with oil-film interferometry is used to investigate the flow field and assess the drag respectively. Results show that the spanwise distribution of the skin friction varies as a consequence of the mean flow heterogeneity and is highly dependent on surface geometry. The swirling strength maps revealed remarkable changes in the secondary flow structures for different ridge shapes. For wide ridges, topological changes occur showing the appearance of tertiary vortices coexisting with the large-scale secondary structures. An imbalance in favour of these tertiary structures occurs over a certain width which take over the secondary structures, causing a swap in the locations of the low- and highmomentum pathways. Furthermore, the results indicate that the spanwise spacing alone is insufficient to characterise the surface heterogeneity. A new parameter $(\xi)$, which is based on the ratio of the perimeter over and below the mean surface height is shown to adequately capture the changes in skin friction and streamwise circulation of the secondary motions. Triple decomposition allowed the quantification of the dispersive stresses for all these cases, which can contribute up to $55 \%$ of the total shear-stress when strong secondary motions occur.

Key words:

\section{Introduction}

Nikuradse (1930)'s seminal work on turbulent flows in non-circular pipes has highlighted the presence of important large-scale features known as secondary flows. These secondary flows embedded within a primary flow manifest in the form of streamwise vortices. Prandtl (1952) showed they can exist in various flow conditions, and classified them into two kinds based on their generation mechanism. The first kind is generated due to mean flow skewness such as in pipe systems with curvatures, whereas the second kind originates from turbulence anisotropy such as in straight non-circular duct flows.

Until the late sixties, the majority of efforts routed towards the description and understanding of these two kinds of secondary flows in the presence of smooth surfaces. The introduction of surface roughness was first probably carried out by Hinze (1967, 1973). Hinze experimentally investigated the turbulent secondary flows in a rectangular

$\dagger$ Email address for correspondence: t.medjnoun@soton.ac.uk 
duct with a partially corrugated wall, and showed the occurrence of secondary flows both in the corners as well as in the rough-to-smooth spanwise step-change. He reported that downwash and upwash motions occurred above the rough and smooth strips respectively.

Ever since, secondary flows caused by surface roughness earned an increased consideration owing to their presence in a wide variety of applications. Their presence is ubiquitous in environmental flows such as fluvial processes, where they are often associated with the formation of bedforms and cause variations in the topographical sediment distribution (Nezu \& Nakagawa 1984; Nezu et al. 1993; Colombini 1993; McLelland et al. 1999; Gyr \& Kinzelbach 2004). In engineering flows, their presence has been observed in ablated turbine blades (Barros \& Christensen 2014), herringbone riblets (Nugroho et al. 2013), superhydrophobic surfaces (Türk et al. 2014), wind farms (Vanderwel et al. 2017) or simply highly ordered roughness (Willingham et al. 2014). In almost all of these examples, when the spanwise characteristic wavelength is comparable to the dominant length scale of the flow, large-scale secondary motions occur (Vanderwel \& Ganapathisubramani 2015; Yang \& Anderson 2017; Chung et al. 2018; Chan et al. 2018). Therefore, the heterogeneity of the flow over a surface depends entirely on the interaction between the surface and the flow developing over it.

These surfaces can be broadly classified into two categories: 1) Surfaces with topographical variation (i.e. adjacent regions of elevations and valleys) and 2) Surfaces with skin-friction variation (i.e. alternating high and low shear-stress). However, it is unclear as to what surface conditions might result in the skin-friction heterogeneity. It is very likely that "roughness" heterogeneity could lead to this condition. Alternately, this can be achieved using strips of different drag increasing/decreasing surfaces. All of these different ways have been observed in the literature (Barros \& Christensen 2014; Nugroho et al. 2013; Türk et al. 2014; Willingham et al. 2014). Irrespective of their type, similar features can be observed over all heterogeneous surfaces. The secondary motions entail a highly three-dimensional flow, with high- and low-momentum pathways (HMP/downwash and LMP/upwash) alternating in the spanwise direction (Mejia-Alvarez \& Christensen 2013). The upwash region harbours large-scale low-momentum regions with strong lateral meandering in the outer region, which induces a vertical tilt in the coherent structures between the HMPs and LMPs, enhancing lateral momentum transfer (Kevin et al. 2017, 2019). These regions are accompanied with a strong Reynolds shear-stress and turbulent activity which are inhomogeneous in the cross-plane. This induces an imbalance between turbulence production and viscous dissipation, which leads to amplified radial fluctuations that translate into streamwise vorticity (Hinze 1973; Anderson et al. 2015; Hwang \& Lee 2018). However, in spite of their impact on the primary flow, the secondary motions are shown to be relatively weak in comparison to the mean flow. In fact, the secondary flows were demonstrated to be simply the result of superimposition of stronger instantaneous vortices, which only occur in a small fraction of the total time, and are unevenly distributed in the cross-plane (Kevin et al. 2017; Vanderwel et al. 2019).

Furthermore, the validity and applicability of established concepts in these flows has been questioned since these concepts were developed for flows over homogeneous roughness. In that regard, the validity of outer-layer similarity hypothesis (Townsend 1976) has been discussed. Prior to the study of Barros \& Christensen (2014), who highlighted secondary motions in a highly irregular surface roughness, Wu \& Christensen $(2007,2010)$ reported statistical as well as structural similarity in the outer layer. They further investigated the inner-outer interactions over the same surface, and reported the amplitude and frequency modulation to be similar to that of a smooth wall at both the HMPs and LMPs, reinforcing the argument of existence of similarity (Pathikonda \& Christensen 2017). Other studies on the other hand reported the absence of outer-layer 
similarity in the mean flow as well as in the turbulence and spectra, owing to roughness effects which extended farther into the outer layer (Yang \& Anderson 2017; Awasthi \& Anderson 2018; Chan et al. 2018; Chung et al. 2018; Medjnoun et al. 2018).

Despite the commonalities between these numerous heterogeneous surfaces, several aspects still remain ambiguous. In the case of surfaces with skin-friction heterogeneity, the upwash and downwash motions systematically occurred above the low and high skin friction, respectively (Hinze 1973; McLelland et al. 1999; Wang \& Cheng 2005; Willingham et al. 2014; Türk et al. 2014; Stroh et al. 2016; Chung et al. 2018). For the topographical heterogeneities, several studies reported the upwash and downwash motions to occur above elevated and recessed regions, respectively (Nezu \& Nakagawa 1984; Wang \& Cheng 2006; Vanderwel \& Ganapathisubramani 2015; Medjnoun et al. 2018; Hwang \& Lee 2018). In contrast, the opposite behaviour was shown in other experimental and numerical investigations (Mejia-Alvarez \& Christensen 2013; Barros \& Christensen 2014; Yang \& Anderson 2017; Awasthi \& Anderson 2018). The reasons for this difference remains unclear since the elevated/recessed regions have been thought of as regions of "high/low" roughness. Additionally, in order to identify the surface properties that characterise best the heterogeneity, many researchers performed parametric studies. It has been shown that the spanwise spacing between adjacent ridges/strips is a relevant surface parameter of the heterogeneity (Vanderwel \& Ganapathisubramani 2015; Yang \& Anderson 2017; Anderson et al. 2018; Chung et al. 2018; Chan et al. 2018). However, other studies have shown that other surface features exhibited a potential to influence the secondary flows, such as the width of the "high" roughness region or the "amount" of roughness (Willingham et al. 2014; Türk et al. 2014; Stroh et al. 2016), or the ridge shape and width (Goldstein \& Tuan 1998; Wang \& Cheng 2006; Hwang \& Lee 2018). Therefore, it becomes natural to look for answers to some of the following questions: How to predict the location of the upwash and downwash motions for the topographical heterogeneous surfaces? What are the other relevant length-scales that carry the heterogeneity information? Can outer-layer similarity still be satisfied in the presence of secondary flows under certain conditions?

The overarching aim of this work is to document the characteristics of these threedimensional flows and address some of the above mentioned questions. We investigate the flow over a smooth ridge-type heterogeneous surface and assess the effect of shape and width of the ridges. Oil-film interferometry (OFI) in conjunction with cross-stream Stereoscopic Particle Image Velocimetry (SPIV) will enable us to assess the impact of these surfaces on the turbulent boundary layer flow. We examine the link between the surface heterogeneity and various other flow quantities, such as local (and span-averaged) skin friction, momentum transport due to turbulent and dispersive stresses, structural similarity as well as boundary layer intermittency. Based on the data, we identify an additional surface parameter that affects the nature of secondary flows. The paper is organised in four sections: The experimental methodology is described in section $\S 2$. The results and discussion are reported in the sections $\S 3$ and $\S 4$, respectively, followed by a summary and conclusions in section $\S 5$.

\section{Experimental methodology}

\subsection{Facility and surface arrangement}

Figure 1 shows the experimental procedure and facility used in the present study. The measurements were carried out in an open-circuit suction wind tunnel at the University of Southampton, in which several rough-wall studies have been documented (Reynolds 

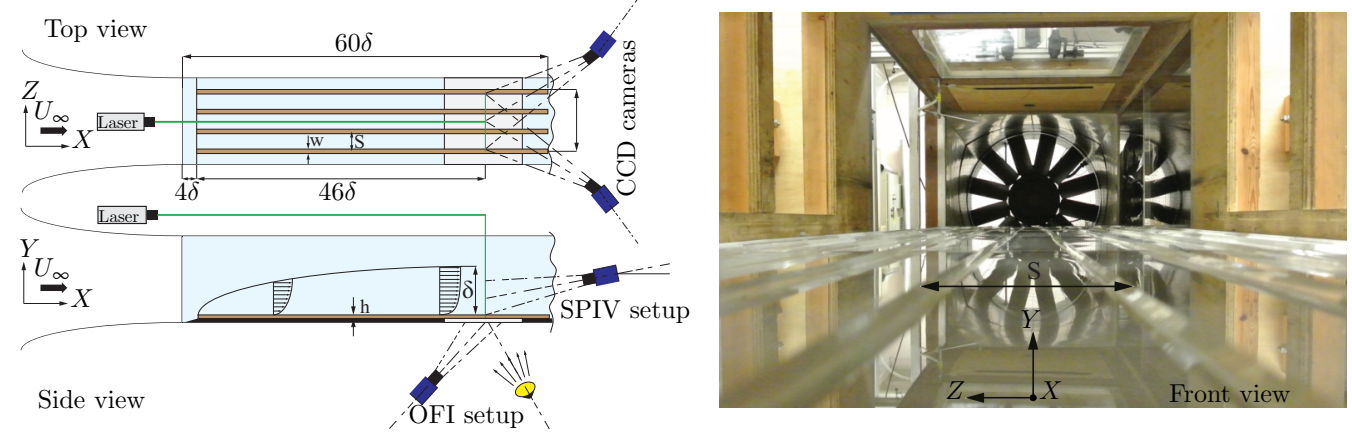

Figure 1: Schematics of the experimental arrangements including the stereoscopic-PIV and the oil-film interferometry set-ups. The measurements of both the OFI and the SPIV are performed at a streamwise distance from the leading edge $x=50 \delta$, with $\delta$ being the spanwise-averaged boundary layer thickness at this fetch.

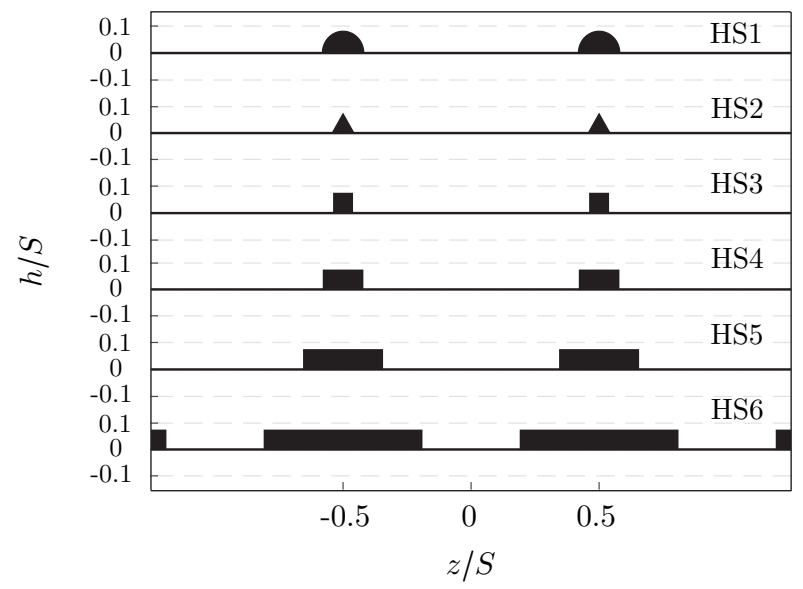

Figure 2: Schematics of the different geometries tested in the present investigation, with the cases HS1, HS2 and HS3 representing the shape effect while HS4, HS5 and HS6 representing the width effect.

et al. 2007; Vanderwel et al. 2017). The extent of the working section is $0.6 \mathrm{~m} \times 0.9$ $\mathrm{m} \times 4.5 \mathrm{~m}$ in $(y, z, x)$; wall-normal, spanwise and streamwise directions respectively. A zero pressure-gradient turbulent boundary layer grows over a flat surface composed by four equally-sized boards; three of which are made of wood over which gloss-PVC plastic sheets are bonded to, ensuring a smooth texture. A fourth board made of clear perspex is used to allow skin-friction measurements using the OFI technique. The boundary layer plates are preceded by a ramp of $0.2 \mathrm{~m}$ long inclined by $4^{\circ}$ to the horizontal ensuring a smooth transition of the flow from the bottom floor of the test section to the boundary layer plates. The flow is tripped using a $6 \mathrm{~mm}$ point-to-point zig-zag turbulator tape of $0.5 \mathrm{~mm}$ height at $0.26 \mathrm{~m}$ downstream the leading edge of the ramp. Further details on how the wind tunnel was operated can be found in a previous study by Medjnoun et al. (2018).

Idealised heterogeneous surfaces were created using different profiles of smooth ridges made of clear perspex (with the same surface texture as the smooth wall). These profiles 
are selected in order to assess the secondary flow sensitivity to shapes and widths. A semi-circle, a triangle and a square are used for the study of the shape effect, while three rectangles with different widths $W$ are used to investigate the width effect. These surfaces will be referred to by their labels HS1 to HS6 as shown in figure 2. Their maximum heights $h$ and spanwise spacings $S$ were chosen to match 0.1 and 1 times the spanwise-averaged boundary layer thickness $\delta$, respectively, in order to maximise potential secondary flows (Vanderwel \& Ganapathisubramani 2015). Details about the geometrical characteristics of the heterogeneous surfaces are summarized in table 1.

\subsection{Oil-film interferometry}

The study of wall-bounded flows requires an accurate assessment of the friction velocity, since most of the similarity analysis of the mean and turbulence quantities depends on the knowledge of this scaling parameter. In this study, we measure this quantity using the oil-film interferometry technique. It is based upon a linear relation between the change in time of the thickness of an oil-film deposited on a test surface on top of which a moving air exerts a tangential force, which is represented by the wall shear-stress.

Since its introduction by Squire (1961) and its application to a flat wall turbulent boundary layer by Tanner \& Blows (1976), many researchers have tested the applicability of the OFI technique under several flow conditions. The method has been used in many flows such as: adverse pressure-gradient boundary layers (Pailhas et al. 2009), turbulent boundary layers subjected to free stream turbulence (Blay Esteban et al. 2017) and also in "rough-wall" boundary layers whose surfaces presented a smooth texture (Akomah et al. 2011; Medjnoun et al. 2018).

Dow Corning 200 Silicone oil droplets are deposited at various locations along the spanwise direction to assess the spanwise variation of the skin friction. Their width is less than $0.1 \delta$ such that they are representative of independent skin-friction measurements. Interferograms are obtained by illuminating the droplets using a sodium lamp with a wavelength of $589 \mathrm{~nm}$. A high-resolution LaVision Imager LX 16 MPixel CCD camera with a Nikon $200 \mathrm{~mm}$ AF Micro lens set at an angle of $25^{\circ}$ from the vertical was fitted to a Scheimpllug adapter to obtain respectively a large field-of-view (FOV) and to satisfy the Scheimpflug condition (shown in the side view of figure 1). A single plane calibration target positioned at the wall in the $(x, z)$-plane was used to determine the mapping function through a third-order polynomial fit. This resulted in a FOV of approximately $0.6 S \times 1.2 S$ in the $(x, z)$-plane. This allowed a simultaneous capture of the development of different spanwise interferograms. A series of 100 images per surface at four different nominal free stream speeds $\left(U_{\infty}=10,15,20\right.$ and $\left.25 \mathrm{~m} \mathrm{~s}^{-1}\right)$ are acquired for approximately 10 minutes in each run using LaVision DAVIS 8.2. The images were subsequently postprocessed using a similar method to that described by Blay Esteban et al. (2017).

One of the main sources of uncertainty in the OFI technique comes from the oil viscosity (Naughton \& Sheplak 2002). Hence, the oil viscosity was calibrated independently of these experiments, using a DHR 3 TA Instruments rheometer for a wide range of temperatures. This allowed a correction of the skin friction estimates caused by variations in temperature during the measurements. Using a linear uncertainty propagation analysis on the wall shear-stress equation from the OFI method (see e.g. Pailhas et al. (2009)), our results highlighted that the leading order error comes from the oil-film viscosity variations. In the present study, the overall relative uncertainty in the skin-friction coefficient accounting for both the systematic and random sources amounts to $3.1 \%$. The average deviation of the current results in $C_{f}$ at matched $\operatorname{Re}_{\theta}\left(\operatorname{Re}_{\theta}=U_{\infty} \theta / \nu\right.$, where $\theta$ and $\nu$ are the momentum thickness and air kinematic viscosity respectively) with the 
smooth wall correlation of Nagib et al. (2007) is less than 2.5\% (see Medjnoun et al. (2018)).

\subsection{Stereoscopic Particle Image Velocimetry (SPIV)}

Following the observations from previous studies, potential secondary flows are expected to generate a large-scale modification of the turbulent boundary layer leading to strong spanwise heterogeneities. Accordingly, the cross-plane $(y, z)$ is investigated using SPIV measurements performed at a streamwise location similar to that of the OFI measurements ( $x=3.2 \mathrm{~m}$ downstream the leading edge) as described in the setup illustrated in figure 1 . The flow is traced by vaporised glycerol-water solution particles generated by a Magnum 1200 fog machine, then illuminated with a laser light sheet sourced by a two-pulse Litron Nd:YAG laser operating at $200 \mathrm{~mJ}$. A LaVision optical system for the beam focus/expansion of the light sheet is used, which comprised of convex and concave lenses in order to focus the beam, and a cylindrical lens in order to expand the sheet with relatively constant thickness in the measurement plane $(\approx 1 \mathrm{~mm}$ thickness $)$. The particle images are recorded by two high-resolution LaVision Imager LX 16 MP CCD cameras fitted with a Nikon $200 \mathrm{~mm}$ AF Micro lens each and mounted on Scheimpflug adapters to account for the oblique view angle $\left( \pm 42^{\circ}\right)$, and are placed at nearly $1 \mathrm{~m}$ from the object plane. A double-sided dual plane calibration target aligned with the laser light sheet was used to determine the mapping function for each camera, using a third-order polynomial fit. This resulted in a FOV of approximately $2 \delta \times 3 \delta$ in the $(y, z)$-plane. Using LaVision's DaVis 8.2 software, 3000 statistically independent realisations of image pairs are acquired for each case at $0.6 \mathrm{~Hz}$, with a time delay between two pulses of $20 \mu \mathrm{s}$ at a free stream velocity $U_{\infty}=20 \mathrm{~m} \mathrm{~s}^{-1}$. This resulted in an average displacement of 7 pixels, which results in roughly $1.5 \%$ bias uncertainty in the measurement (given the subpixel uncertainty being around \pm 0.1 pixels). The velocity vector fields were subsequently obtained by interrogating particle images using a decreasing multipass scheme starting from window sizes of 48 pixels $\times 48$ pixels to a final pass at 24 pixels $\times 24$ pixels with $50 \%$ overlap. The resulting vector fields were subsequently low-pass filtered at $l_{S P I V}^{+} \approx 55$ (with $l_{S P I V}^{+}$being the inner-normalised interrogation window size of the SPIV grid). This prevents the comparison between cases to be affected by the grid resolution due to marginal differences in $\operatorname{Re}_{\tau}\left(R e_{\tau}=U_{\tau} \delta / \nu\right.$, where $U_{\tau}$ is the friction velocity).

\section{Results}

\subsection{The mean viscous drag and flow topology}

Results from the OFI measurements are presented in figure 3. They describe the response of the surface shear-stress to the presence of surface heterogeneity at various moderate Reynolds numbers $R e_{x}\left(R e_{x}=U_{\infty} x / \nu\right.$, where $x$ is the distance to the measurement location from the leading edge) obtained by varying the freestream speed. We specifically quantify the amount of viscous drag experienced by the wall at the valley, since this information is available for all the cases, estimated by:

$$
C_{f}^{*}=\left\langle C_{f}\left(z_{i}\right)\right\rangle_{W_{v}},
$$

with $\langle\cdot\rangle_{W_{v}}$ being the spatial average at the valley width $\left(W_{v}=S-W\right)$ across the different discrete points $z_{i}$ where the skin-friction coefficient was measured. The results show clearly the decay of the skin friction with respect to Reynolds number. This indicates that regardless of the surface heterogeneity, the flow remains Reynolds number dependant as opposed to rough-wall turbulence which asymptotes towards a constant value of $C_{f}$ at 


$\begin{array}{lccccccccccc}\text { Case } & \text { Symbol } & h / \delta & S / \delta & W / \delta & (S-W) / \delta & C_{f}^{*} \times 10^{3} & C_{F} \times 10^{3} & \beta^{*} & R e_{\tau} & \Gamma^{*} \times 10^{2} & \xi \\ \text { HS1 } & \bullet & 0.09 & 1.17 & 0.17 & 1 & 3.01 & - & 1.16 & 3125 & 4.42 & 4.61 \\ \text { HS2 } & \Delta & 0.08 & 1.21 & 0.08 & 1.12 & 3.19 & - & 1.23 & 3347 & 5.27 & 6.83 \\ \text { HS3 } & \square & 0.09 & 1.14 & 0.08 & 1.05 & 2.82 & - & 1.09 & 3135 & 3.74 & 4.62 \\ \text { HS4 } & \diamond & 0.09 & 1.26 & 0.18 & 1.07 & 2.88 & 2.87 & 1.11 & 2828 & 3.75 & 3.74 \\ \text { HS5 } & \text { ఓ } & 0.09 & 1.26 & 0.35 & 0.90 & 2.72 & 2.80 & 1.05 & 3239 & 3.21 & 2.71 \\ \text { HS6 } & \text { i } & 0.08 & 1.20 & 0.67 & 0.52 & 2.38 & 2.76 & 0.92 & 2829 & 2.78 & 1.72\end{array}$

Table 1: Geometrical characteristics and the aerodynamic parameters for the different heterogeneous surfaces measured at $R e_{x} \approx 4 \times 10^{6}$. The parameters $C_{f}^{*}, C_{F}$ and $\beta^{*}$ represent measures of the skin-friction coefficient from the OFI measurements and are discussed in $\S 3.1$. The parameters $\Gamma^{*}$ and $\xi$ represent the secondary flow circulation and the surface parameter respectively and are discussed in $\S 3.3$. The dimensions of $h$ and $S$ were fixed to 6 and $80 \mathrm{~mm}$ respectively. The spanwise-averaged boundary layer thickness $\delta$ was measured by identifying the wall-normal distance at which the spanwise-averaged streamwise velocity reached $99 \%$ of the free stream speed $U_{\infty}$

"high-enough" Reynolds numbers (Jiménez 2004; Shockling et al. 2006; Flack \& Schultz 2014; Djenidi et al. 2018). This can be explained by the fact that these surfaces do not present streamwise rough protrusions which in addition to viscous drag, lead to pressure drag which cause the fully-rough behaviour (Napoli et al. 2008; Yuan \& Piomelli 2014). This observation leads to hypothesise that these smooth heterogeneous surfaces (as long as their texture is smooth) cannot meet the fully-rough conditions, and therefore will always remain Reynolds number dependant (from the skin friction perspective). Meanwhile, these results show that the skin-friction coefficient is also dependant on the surface heterogeneity condition. In fact, if we compare the skin-friction coefficients at matched $R e_{x}$, we can clearly distinguish that the triangular cross-section case produces more skin friction than the half-round and the square, despite them having the same valley width $((S-W) / \delta \approx 1)$. This indicates the effect of geometry on the mean streamwise velocity gradient at the wall.

Schlichting's power-law curve (Schlichting 1979) is shown to be in a good agreement in comparison with the present smooth-wall baseline case (compare the solid line against the blue right-pointing triangle in figure 3). The power-law further allowed to quantity these relative changes against the smooth wall, and are tabulated in table 1 under the label $\beta=\left(C_{f}^{*} / C_{f_{\text {smooth }}}\right)$. This quantity represents a measure of the relative drag change at the valley due to the surface heterogeneity. It indicates a net increase in skin friction at the valley for the cases HS1 to HS4 (relatively matched valley width $(S-W) / \delta$ ), with a $23 \%$ increase for HS2. On the other hand, HS5 and HS6 recorded lower values especially for the HS6 case which shows local skin-friction reduction. This can be due to the strong viscous blockage induced by the interaction of two boundary layers, one on the either ridge's side walls. However, if we compare the spanwise-averaged skin-friction coefficient $C_{F}$ (also tabulated in table 1), we notice that the overall drag is still higher than that of a smooth wall at an equivalent Reynolds number $R e_{x}$. Even though the latter information is not available for the first three cases since OFI method cannot be applied above the ridges, the expected overall drag should also be higher than that of a smooth wall. From the above results, it is clear that the mean wall shear-stress depends 


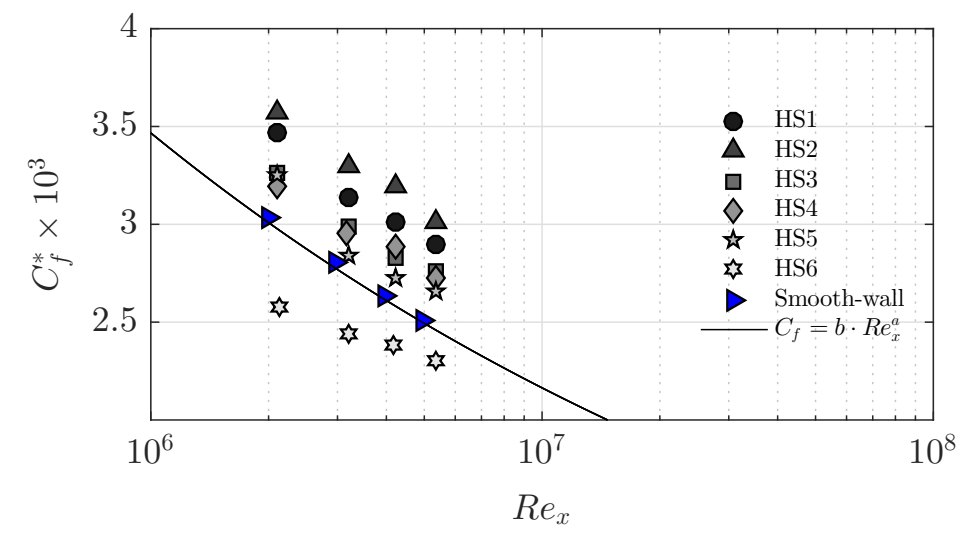

Figure 3: Variation of the valley spanwise-averaged skin-friction coefficient at various moderate Reynolds numbers for the different heterogeneous surfaces. The black solid line represents Schlichting's power-law with $a \approx-1 / 5$ and $b \approx 0.058$. The blue right-pointing triangles represent the smooth-wall data from Medjnoun et al. (2018).

on both the geometry of the surface heterogeneity and Reynolds number. This can be further explored by examining the mean flow using the SPIV data.

Figure 4 shows the normalised mean streamwise velocity maps and the spanwise distribution of the skin-friction coefficient, with figures $4(a, b, c)$ delineate the shape effect while figure $4(\mathrm{~d}, \mathrm{e}, \mathrm{f})$ describe the width effect. Consistent with previous studies, strong spanwise heterogeneities in the mean flow can be distinguished. These are represented in the form of alternating high- and low-momentum pathways between valleys and peaks respectively. The degree of spanwise heterogeneity in the mean flow has also impacted the spatial distribution of the skin-friction coefficient, due to the spanwise variation in the mean velocity gradient at the wall. This is in line with our previous experimental study (Medjnoun et al. 2018) and the direct numerical simulation (DNS) reported by Hwang \& Lee (2018), which showed that the spanwise distribution of the skin friction to strongly vary across the span, especially near the spanwise step-change with sharp changes occurring due to the discontinuity (see figure 11 of Hwang \& Lee 2018).

Figure 4 also shows relative similarities between the mean velocity maps and the spanwise distribution of $C_{f}$ for HS1 to HS4. In fact, despite the inability of measuring the local skin friction at the peak symmetry plane $(z / S= \pm 0.5)$, the shape of $C_{f}(z)$ remains reasonably similar except for the change in magnitude. It appears that the maximum skin friction in the valley occurs at its symmetry plane $(z / S=0)$, which then decreases in the vicinity of the ridge. This observation is also consistent with the DNS results of Hwang \& Lee (2018) for the ridges of smaller widths with large spanwise spacings. The mean flow topology and the corresponding skin-friction distribution presented in figure 4 show a good correlation, especially for the cases HS1 to HS4. In fact, regions of constant skin friction are accompanied with a locally homogeneous mean flow (HMP), while regions of high gradient in the skin friction are associated with strong mean flow heterogeneity (LMP and HMP boundaries).

The topology of the mean flow starts to exhibit differences for the case of HS5 and changes drastically for HS6. The LMPs appear to widen for HS5 above the ridge, while the HMPs are narrower in the valley. This is accompanied by an increase in skin friction above the ridge when compared to HS4, while the average skin friction in the valley decreases compared to HS4. In the case of HS6, the increase of the ridge width has 


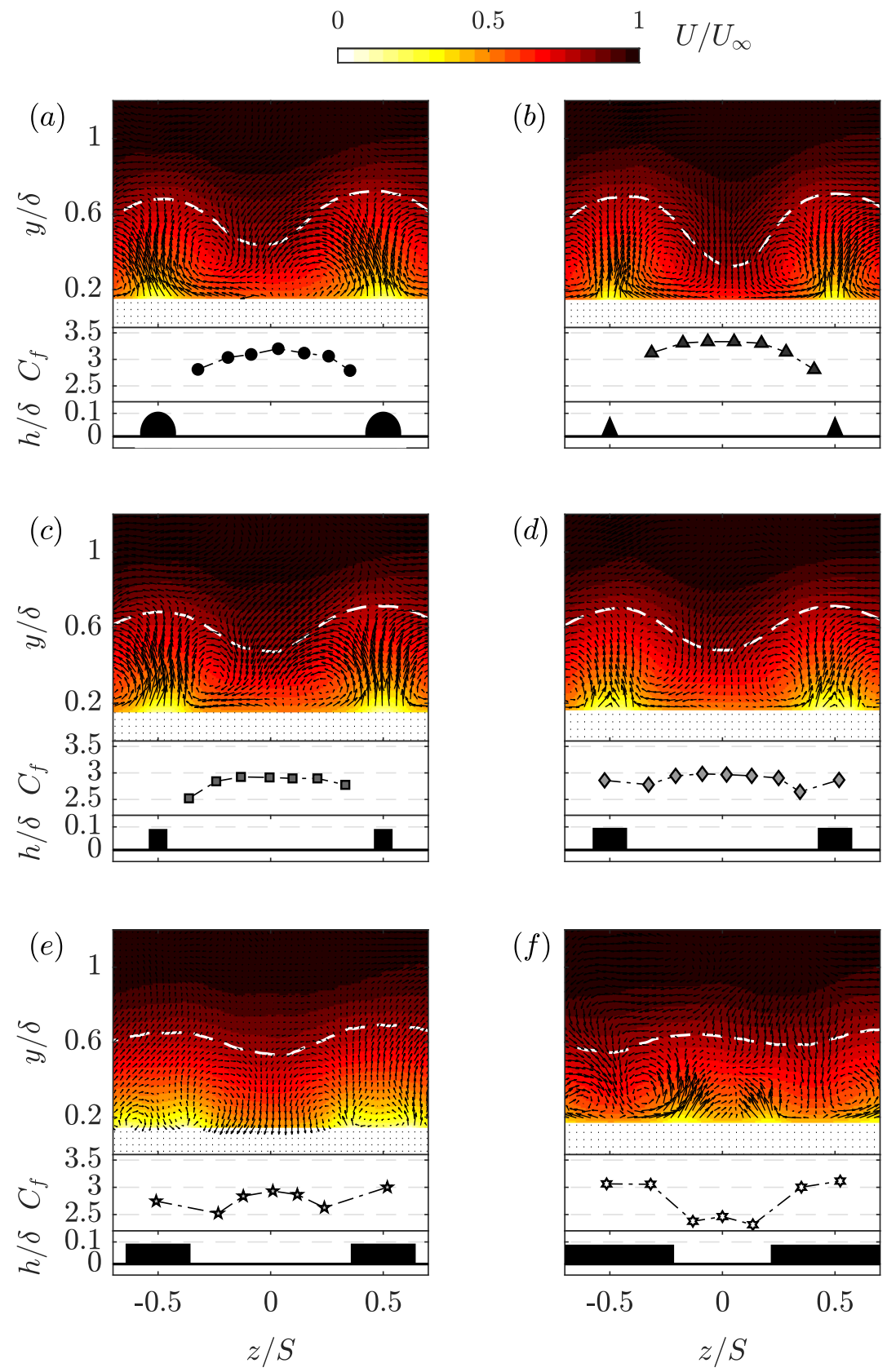

Figure 4: Contour maps of the normalised mean streamwise velocity and the spanwise distribution of the skin-friction coefficient at $R e_{x}=4 \times 10^{6}$. (a): HS1, (b): HS2, (c): HS3, $(d)$ : HS4, $(e)$ : HS5, $(f)$ : HS6. The cross-sections of the roughness geometries are included at the bottom of the figures to scale for reference. The white dashed-line represents the velocity contour level $U=0.9 U_{\infty}$. The mean in-plane velocity vector plot $V$ and $W$ are superimposed to highlight the vortical structures. 
led to a reorganisation of the flow topology, clearly showing a swap in the locations of the HMPs and the LMPs. This rearrangement in the mean flow has also impacted the spanwise distribution of the skin-friction coefficient, as higher skin friction is now recorded above the ridge while reduction in skin friction is observed in the valley, causing the local drag reduction. This can be explained by an increase in the viscous blockage caused by the ridge side wall boundary layers interacting with one another. This seems to specifically occur when the ridges are made wide enough such that they start influencing the neighbouring ones. It is also of interest to point out that for HS5 and HS6 that the degree of spanwise mean flow heterogeneity is lower than that of the other cases. As it can be seen from figures $4(\mathrm{e})$ and (f), the spanwise variation in the mean velocity contour level $0.9 U_{\infty}$ highlighted with the white dashed-line (or the levels seen beyond $0.6 \delta$ ) is relatively weaker when compared with the rest of the cases.

The results shown in the figures $4(\mathrm{a}-\mathrm{e})$ are all in agreement with the previous studies that showed the HMPs and LMPs to occur over recessed and elevated regions respectively (Nezu \& Nakagawa 1984; Colombini 1993; Wang \& Cheng 2006; Vanderwel \& Ganapathisubramani 2015; Medjnoun et al. 2018; Hwang \& Lee 2018). However, the result shown in figure 4(f) seems more to line up with the results of Barros \& Christensen (2014), Yang \& Anderson (2017) and Awasthi \& Anderson (2018). These findings indicate that for ridge-type heterogeneous surfaces with $S / \delta \sim \mathcal{O}(1)$, both scenarios can occur. Nonetheless, these differences in observations are likely to stem from an additional length scale at play besides $S / \delta$. This leads us to further question how are these observed differences related to the characteristics of the large-scale secondary flows.

\subsection{Effect on the upwash and downwash of the secondary flows}

The effect of surface conditions on the upwash/downwash is shown in the mean wallnormal velocity component in figure 5. This component is of relevance since it directly assess quantitatively and qualitatively the effect of ridge geometry on the upwash and downwash motions, besides being a constitutive component of the streamwise vorticity. Figure 5 shows the mean wall-normal velocity maps for five cases out of six (HS5 is omitted for brevity) in the left panel, while contour levels of the ridge zoomed-in are presented to the right. These maps clearly illustrate both the effect of shape and width on the magnitude and distribution of the upwash/downwash motions. The top three maps (left and right) indicate the presence of three different ways of upwash motions that depend on shape. For HS1, the strongest upwash point seems to occur at about $2 h$, whereas for HS2 and HS3 the strongest upwash points seem to be fixed very close to the ridge corner(s), with similar attributes to that of an external flow over a smooth corner chine (Moinuddin et al. 2004).

Figure 5 indicates that both the magnitude and distribution of upwash can be related to the cross-section (curvature) of the ridge. HS2, which shows the most noticeable upwash, has its side walls in a configuration that promotes merging of the flow being deflected to make up a strong single upwash region. Whereas the convex nature of the cross-section in HS1 and the presence of a finite width between the two vertical side walls of HS3 can create more resistance to the flow which is moving radially towards the centre of the ridge, resulting in a reduction in the strength of the upwash motion. Other studies have also shown that the deflection of the spanwise motions due to the resistance created by the surface elevations is detrimental in the process of the secondary flow generation (Goldstein \& Tuan 1998; Wang \& Cheng 2006; Hwang \& Lee 2018).

For the width effect cases, we observe that the upwash regions for HS4 and HS6 become more distinct, however tend to decrease in strength. This is further emphasised by looking at the ellipsoid at a matched contour level across cases $\left(\mathrm{V}=1 \% U_{\infty}\right)$. The 


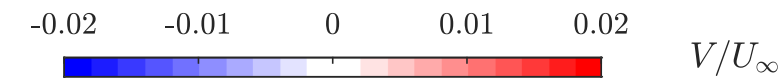

(a)

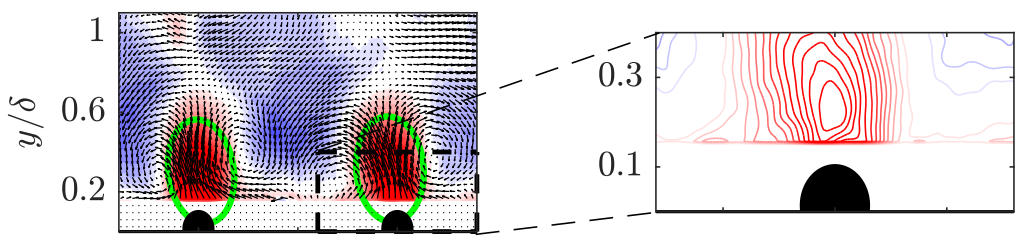

(b)

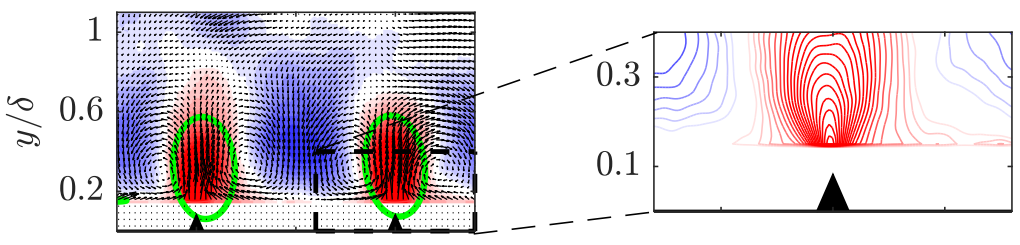

(c)

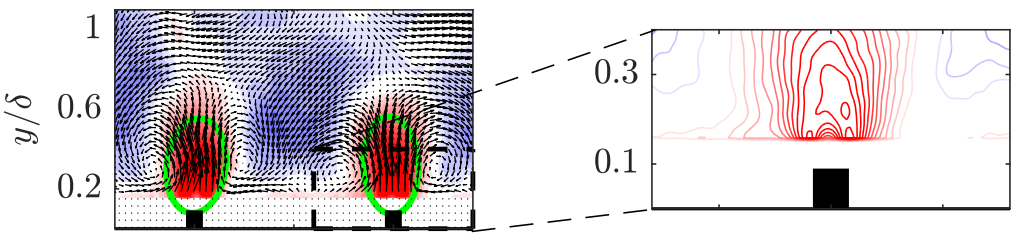

(d)

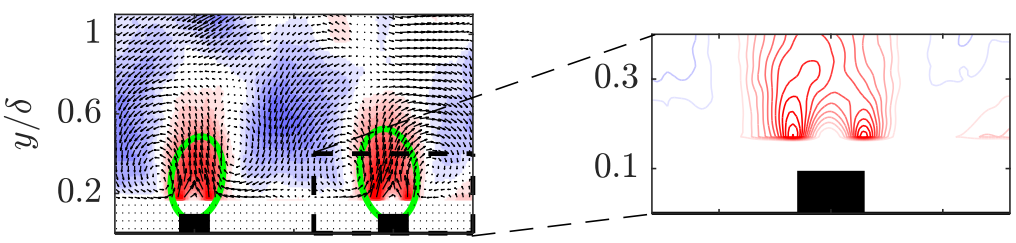

$(f)$

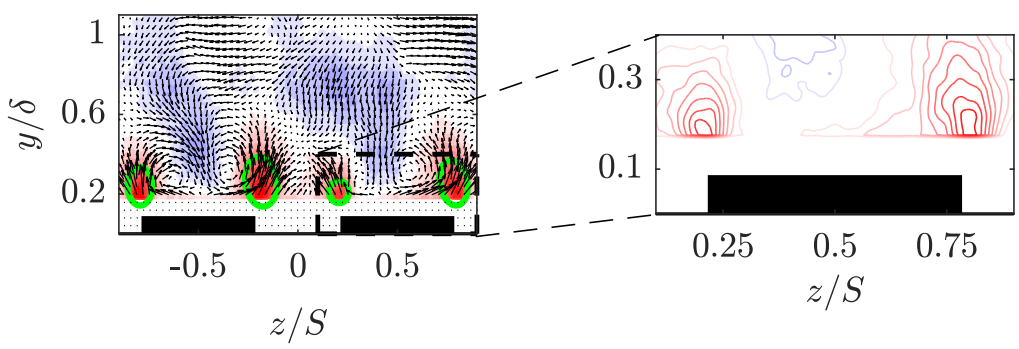

Figure 5: Effect of the roughness geometry on the topology of the mean vertical velocity component (left panel) with a zoom-in of the upwash region (right panel). (a): HS1, (b): HS2, $(c)$ : HS3, $(d)$ : HS4, $(e)$ : HS6. The mean in-plane velocity vector plot $V$ and $W$ are superimposed to highlight the vortical structures. The green contour level represents $V=1 \%$ of $\left.U_{\infty}\right)$.

contour level seems to encapsulate a relatively larger area for HS1 to HS3, then starts decreasing for wider ridges. This eventually ends up in a formation of two distinct lumps of upwash regions over a single ridge. It follows that these distinct upwash regions start interacting with the neighbouring ones to eventually end up forming a new coherent upwash region, leading to the swap in the LMP and HMP locations observed in figure $4(\mathrm{f})$. It is also important to point out that if wider ridges were to be used $\left(W_{v} \ll h\right)$, these upwash/downwash motions would essentially die out, as the surface will recover 

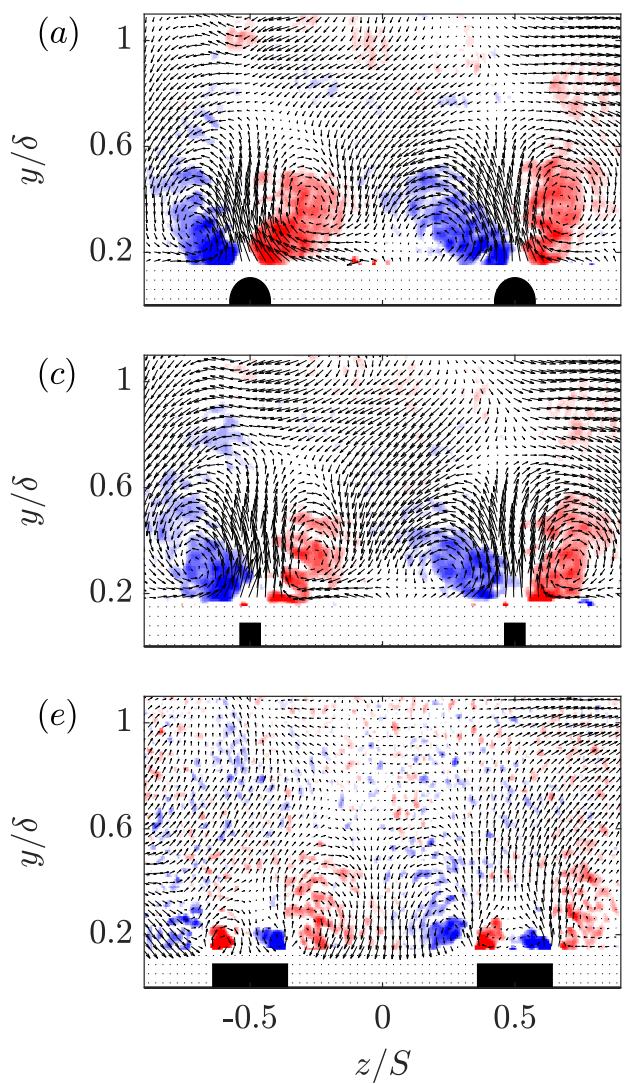

(b)

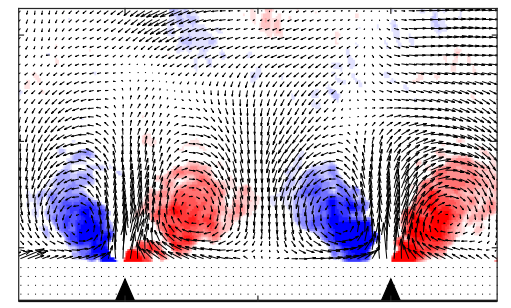

(d)

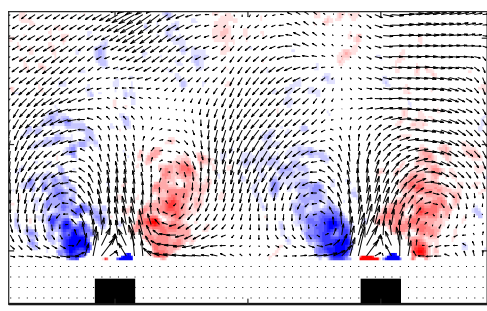

$(f)$

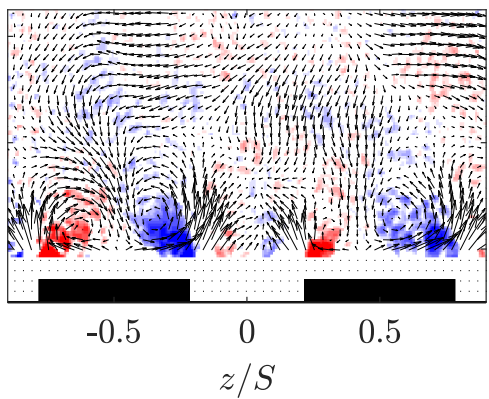

Figure 6: Effect of the heterogeneous surfaces on the normalised vorticity-signed swirling strength. $(a)$ : HS1, $(b)$ : HS2, $(c)$ : HS3, $(d)$ : HS4, $(e)$ : HS5, $(f)$ : HS6. The mean in-plane velocity vector plot $V$ and $W$ are superimposed to highlight the vortical structures on top of the swirling motions.

homogeneity. Given the changes observed in the mean wall-normal velocity component due to the different types of ridges, it is essential to also look at how the secondary motions are distributed in these flows.

Following the aforementioned changes observed in the mean flow topology, proportional changes in the generated streamwise vorticity are to be expected. The identification of these streamwise time-averaged vortices was achieved through the determination of the vorticity-signed swirling strength $\lambda_{c i}$. This quantity is expressed as the imaginary part of the complex eigenvalue of the cross-plane velocity gradient tensor, multiplied by the sign of the vorticity in order to identify the rotation sense of the streamwise vorticity (see e.g. Anderson et al. (2015)). The results are shown in figure 6 and indicate largescale secondary flows associated with the different heterogeneous surfaces. Consistent with previous studies, these secondary flows consist of a pair of counter-rotating vortices formed on the top of either sides of the surface elevations. However, distinctive characteristics are shown across cases, both related to magnitude and distribution of these largescale vortices. Globally, the spatial extent of $\lambda_{c i}$ for the HS1-HS3 cases is qualitatively similar, although its magnitude seems to be affected by the ridge geometry. However, 
remarkable differences are highlighted for the cases HS4-HS6. In fact, changes in the streamwise vorticity start to occur; as the ridges become wider for a fixed $S / \delta$, a pair of new small counter-rotating vortices of opposite sign are visible above the ridges. Similar additional vortices were also observed by Vanderwel \& Ganapathisubramani (2015) but it was speculated that these could have been caused by the presence of small pins on the top of the LEGO brick roughness. However, in a more recent study by Vanderwel et al. (2019) along with the DNS of Hwang \& Lee (2018), these were reported to exist even for a smooth flat ridge, identical to the HS4-HS6 cases. The results are similar from both the present experiment and the DNS, and these new structures are shown to grow stronger for wider ridges, while a weakening of the previous large-scale vortices is observed. This feature is believed to also be present for HS3 despite not evident from the map, as it is too small to be captured with the current measurement. Other recent studies also reported these additional streamwise vortices over different surface conditions, terming them tertiary flows to differentiate them from the initial large-scale vortices identified as secondary motions (see e.g. Goldstein \& Tuan 1998; Türk et al. 2014; Stroh et al. 2016; Benschop \& Breugem 2017). However, it should be noted that according to Prandtl's definition, any motion occurring in the cross-plane perpendicular to the primary flow should be regarded as a secondary motion/flow. Hence, these different structures can be interpreted as manifestations of different topological states of a secondary flow. In the present description, the terms "secondary" and "tertiary vortices" will be used to identify the previously large-scale (side ridge) and newly (above ridge) streamwise vortices.

As highlighted in figure 6, these secondary and tertiary vortices are shown to have opposite signs, while their size and to a lesser extent magnitude seem controlled by the valley width $(S-W) / \delta$ (also shown in the figures 8 and 9 of Hwang \& Lee 2018). It appears that in the vertical direction, the secondary structure propels the low-speed fluid above the ridge away from the wall, and brings the high-speed fluid from the free stream towards the valley. The tertiary structure on the other hand, attempts to bring the high-speed fluid from the free stream down to the ridge. This is accompanied with a radial motion from the tertiary structure that pushes the low-speed fluid above the ridge towards the valley. Similarly in the spanwise direction, the secondary vortices drive the high-speed fluid from the valley towards the ridges, opposing the tertiary ones. Beyond a given width, the extent of the streamwise vorticity changes due to an imbalance in favour of the tertiary structures which overcomes the secondary vortices. The side ridge secondary vortices continue to weaken leading to the observed swap of the LMP and the HMP locations. At this stage, the tertiary structure becomes the main feature in the cross-plane, resulting in a secondary motion/flow which predominantly produces downwash over the ridges and upwash in between. However, this phenomenon can only take place for spanwise heterogeneous surfaces whose ridges allow the generation of two distinct upwash regions (for example rectangular shapes). In fact, it is difficult to imagine this phenomenon occurring for HS1 and HS2 regardless of their widths.

\subsection{Relation between the wall shear-stress, secondary flows and surface geometry}

It is reasonable following the above observations to investigate a potential correlation between the surface heterogeneity, the magnitude of the secondary motions as well as the amount of drag caused by these surfaces. While it can be straightforward to find a metric to quantify the strength of the secondary motions (e.g. cross-flow turbulent kinetic energy or streamwise circulation), it is less clear which parameters should be used to characterise the surface geometry. For homogeneous rough surfaces, there are abundant surface parameters in the literature that are used to correlate the surface and aerodynamic properties of the flow (Napoli et al. 2008; Flack \& Schultz 2010; Placidi 
\& Ganapathisubramani 2015; Thakkar et al. 2017). Whether it is randomly arranged, highly ordered or irregularly distributed roughness, it can have strong dependence on the amplitude, spacing, density, slope or directionality among other parameters. There is clearly no consensus for a generic formula that will eventually represent all the surfaces.

It has been suggested that for secondary motion producing surfaces, the spanwise wavelength scaled with the dominant length scale of the flow $(S / \delta)$ is a good parameter (Vanderwel \& Ganapathisubramani 2015; Yang \& Anderson 2017; Chung et al. 2018; Chan et al. 2018). It was also argued that for the ridge-type heterogeneous surfaces, the valley width $(S-W) / \delta$ is important as it can substantially increase or reduce the strength of the secondary motions (Hwang \& Lee (2018)). Similarly, for the strip-type heterogeneity, Willingham et al. (2014) have shown that the high-roughness width can either increase or decrease the intensity of the upwash/downwash motions by influencing the strength of the transverse mixing. In the present study, most of these scaling parameters would fail to capture the trends exhibited by the secondary flow. In fact, with $S / \delta$ being constant throughout all the cases, $(S-W) / \delta$ constant for the HS2-HS4 cases and the effective slope argument being constant for the HS3-HS6 cases, we need to adopt a different parametrisation since the previous ones cannot adequately represent all these flow changes.

The relations between the strength of these secondary flows and both the surface condition and the spanwise-averaged wall shear-stress are presented in figure 7 . Figures $7(\mathrm{a})$ and (b) quantify the variation in the streamwise circulation $\Gamma^{*}$ and the skin-friction coefficient $C_{f}^{*}$ produced by these surfaces at the valley (since this estimate is available for the different test cases) with respect to the surface heterogeneity parameter $\xi$. Lastly, figure $7(\mathrm{c})$ shows the variation of $C_{f}^{*}$ with respect to $\Gamma^{*}$. The quantities $\Gamma^{*}$ and $\xi$ which are tabulated in the table 1 are defined as

$$
\begin{gathered}
\Gamma=\int_{0.2 \delta}^{\delta} \int_{-S / 2}^{S / 2}\left|\Omega_{x}\right| d z d y, \\
\xi=\frac{A_{v}}{A_{p}}, \quad \text { where }\left\{\begin{array}{l}
A_{v}=\int_{-S / 2}^{S / 2} \sqrt{\left(1+\left(\frac{\partial h}{\partial z}\right)^{2}\right)} d z, \text { for } h \leq \bar{h}, \\
A_{p}=\int_{-S / 2}^{S / 2} \sqrt{\left(1+\left(\frac{\partial h}{\partial z}\right)^{2}\right)} d z, \text { for } h>\bar{h},
\end{array}\right.
\end{gathered}
$$

with $\Gamma^{*}=\Gamma /\left(\delta U_{\infty}\right)$ representing the normalised circulation of the streamwise vorticity $\Omega_{x}$. In this study, $\Gamma^{*}$ essentially quantifies the amount of streamwise vorticity produced in the outer region of the turbulent boundary layer (above $0.2 \delta$ ). On the other hand, $\xi$ represents an attempt to parametrise the surface using a ratio of the recessed (valleys) and elevated (peaks) wetted area. Since the surfaces are streamwise homogeneous, only the perimeters are considered in this study, and are defined as recessed $\left(A_{v}\right)$ and elevated $\left(A_{p}\right)$ areas whenever below or above the mean roughness height $\bar{h}$, respectively. As shown in figures $7(\mathrm{a})$ and (b), both the amount of streamwise vorticity and frictional drag are affected by these heterogeneous surfaces, despite them having the same $S / \delta$. This result provides a justification for this surface parametrisation since both width as well as shape of the ridges are captured by $\xi$.

In figure $7(\mathrm{a})$, the streamwise circulation is shown to vary almost linearly within the range of $\xi$ tested herein. If we consider the asymptotic limits of $\xi$, two behaviours are expected to take place: (i) for $\xi \rightarrow 0$ the secondary flows are expected to be small enough such that they are confined in the roughness sublayer and the flow will essentially recover homogeneity in the mean and turbulent sense $\left(\Gamma^{*} \rightarrow 0\right)$. This is the limit where the surface is homogeneous. (ii) for $\xi \rightarrow \infty$, this can be thought of as a transitional 

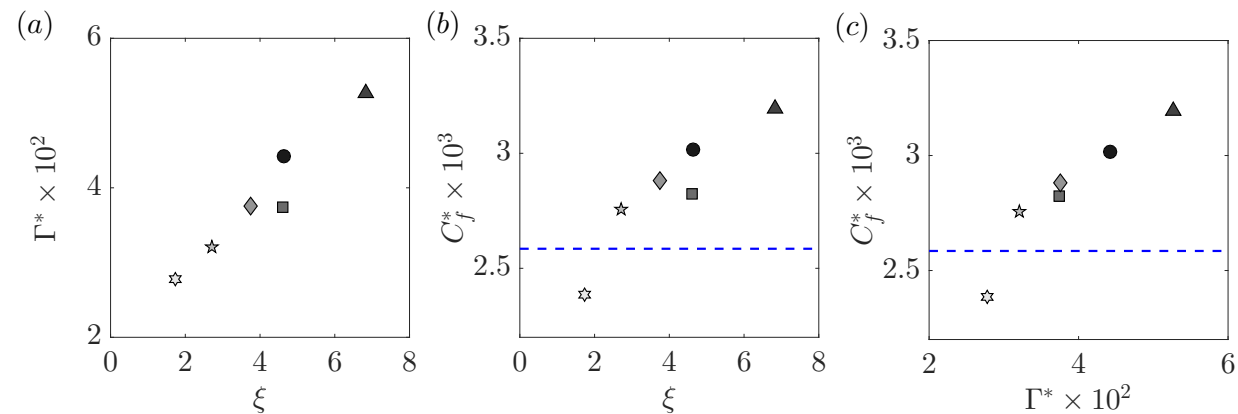

Figure 7: (a) Variation of the normalised compensated circulation $\Gamma^{*}$ and $(b)$ the valley spanwise-averaged skin-friction coefficient with respect to the surface parameter $\xi$. (c) The effect of the secondary flow circulation on the drag experienced at the valley. The blue dashed-line represents the smooth-wall skin-friction coefficient at an equivalent $R e_{x}$. See table 1 for the symbol legend.

surface that will result in localised but large secondary flows over/near the ridges, while farther away from them, the flow is expected to remain homogeneous. In this case, the integrated circulation $\Gamma^{*} \rightarrow \Gamma_{\infty}^{*}$. Between these two limits, a third region can be identified as $a<\xi<b$, where $a$ and $b$ representing the lower and upper limits. This region translates to a strongly heterogeneous flow regime with possible topological bifurcations of the streamwise rollers, with the occurrence of tertiary or even quaternary structures depending on the ridge geometry (Hwang \& Lee 2018). This results in a highly threedimensional flow which leads to the observed increased circulation $\Gamma^{*}$ and drag. These observations are also in line with the study carried out by Yang \& Anderson (2017), which provided similar evidence using a comprehensive range of values of the ridge spanwise spacing normalised with the channel half-height. Their LES results revealed that the spanwise spacing scaling indicates when the flow is expected to become homogeneous, heterogeneous or transitional supporting the idea of existence of three distinct regimes. In their study, these bands were termed roughness, intermediate and topography for homogeneous, heterogeneous and transitional respectively. Similar observations have also been made clear for the alternating high and low shear-stress heterogeneity with the recent direct numerical simulations of Chung et al. (2018).

Furthermore, figure 7(b) shows that the local (valley spanwise-averaged) drag increases with increasing $\xi$. While a drag reduction is observed for HS6, $C_{f}^{*}$ increases albeit with a lower rate when increasing $\xi$. For $\xi \rightarrow 0$, the valley skin friction $C_{f}^{*}$ is naturally expected to tend to 0 . However, if we considered the total drag $C_{F}$, it might be similar to the homogeneous case (or higher), which in this case is highlighted by the blue dashed-line. It is interesting to note that it might be possible to find a surface geometry that leads to an optimal secondary flow condition that would reduce the spanwise-averaged wall shearstress. Further studies are necessary to examine the feasibility of such surfaces and is beyond the scope of the present work. If $\xi \rightarrow \infty$, the skin friction is also expected to return to the homogeneous case at some point since the secondary motions will be significantly localised. However, how/when this would happen is also open for further exploration. Overall, we observe that changes in streamwise circulation induce proportional changes in the wall shear-stress as demonstrated in figure $7(\mathrm{c})$ (and vice-versa). It essentially reveals that a possible linearity can exist for strong/large secondary flow cases, with an increase in the streamwise circulation leading to a proportional increase in the wall shear-stress. 


\subsection{Effect of surface heterogeneity on the turbulence properties}

The impact of the surface heterogeneity on the turbulence organisation is further analysed using a triple decomposition performed on the three component velocity vector in the cross-plane. This concept has been well documented and widely used to assess flows presenting spatial inhomogeneities (Reynolds \& Hussain 1972; Raupach \& Shaw 1982; Nikora et al. 2007). In this framework, the velocity field can be expressed as

$$
u_{i}(y, z, t)=U(y)+\tilde{u}(y, z)+u(y, z, t)
$$

where $u_{i}$ is the instantaneous velocity field measured at a given streamwise location, $U(y)$ is the spatial- and time-averaged velocity profile, $\tilde{u}(y, z)$ is the spatial deviation field independent of time and $u(y, z, t)$ is the time fluctuating part from the Reynolds double decomposition. This decomposition allows us to quantify the amount of stresses arising from the mean flow heterogeneity, which contribute to the total stresses as forminduced (dispersive) stresses. In the case of strong spatial heterogeneities, these dispersive stresses can play a major role in the transport of momentum flux, which can impact the organisation of the turbulent boundary layer structure (Türk et al. 2014; Meyers et al. 2019).

Comparative maps of the turbulent, dispersive and total shear stresses denoted $\overline{u v}, \overline{\tilde{u} \tilde{v}}$ and $\overline{\tau_{x y}}$ respectively are presented in figure 8 (in columns), for different cases (in rows). As expected, the dispersive stresses are not negligible with respect to the turbulent ones. In fact, they appear to be non-zero for nearly two thirds of the extent of the boundary layer thickness with varying intensity along the spanwise direction (shown in the second column from the left of figure 8). The patterns of the turbulent and dispersive shear stresses do not show particular resemblance. In fact, while the overall distribution of $\overline{u v}$ seems to follow a similar wavy character as the mean flow, $\overline{\tilde{u}} \tilde{v}$ on the other hand appears to be more localised. The dispersive shear-stress is shown to have high intensity above the surface elevation (especially near the ridge edges) as opposed to a weaker magnitude recorded at the valley. Away from the ridges, $\overline{\tilde{u}} \tilde{v}$ is shown to span nearly half of the valley extent with highest intensities being recorded at nearly $0.3 \delta$ from the wall. Nevertheless, the dispersive shear-stress is shown to vanish between the peaks and valleys, whose regions are characterised by enhanced spanwise and weak wall-normal motions. These observations appear to mainly apply for the cases HS1-HS4. This is in contrast with HS5 and HS6, which were shown previously to be the least heterogeneous cases. As illustrated in middle figures $8(\mathrm{e})$ and (f), $\overline{\tilde{u} \tilde{v}}$ is observed to reduce in spatial extent and strength, where only a smaller dispersive shear-stress remains above the ridges especially for HS6.

For $\overline{u v}$, a relatively weaker magnitude of the stress is observed at the valley in comparison to the ridge, with an overall distribution being similarly modulated as the mean flow. However, this magnitude is expected to increase when approaching the near-wall region below the currently measured FOV, especially in the vicinity of the centre of the valley. We can also observe from figure 8 that despite the different ridge geometries for the cases HS1-HS4, the turbulent shear-stress remains globally similar between cases. This observation can perhaps be explained by the fact that once these large-scale secondary motions are formed, changes in surface heterogeneities result only in variations of their strength, without necessarily affecting their spatial extent. This mostly leads to an increased dispersive component, while the turbulent shear stresses remain relatively unchanged. However this is believed to be only valid when a strong degree of heterogeneity exists. This is further substantiated from the total shear-stress maps $\overline{\tau_{x y}}$ shown in the third column from the left of figure 8 . They mainly show that for large-scale secondary flows, their resulting total stresses remain relatively unchanged 

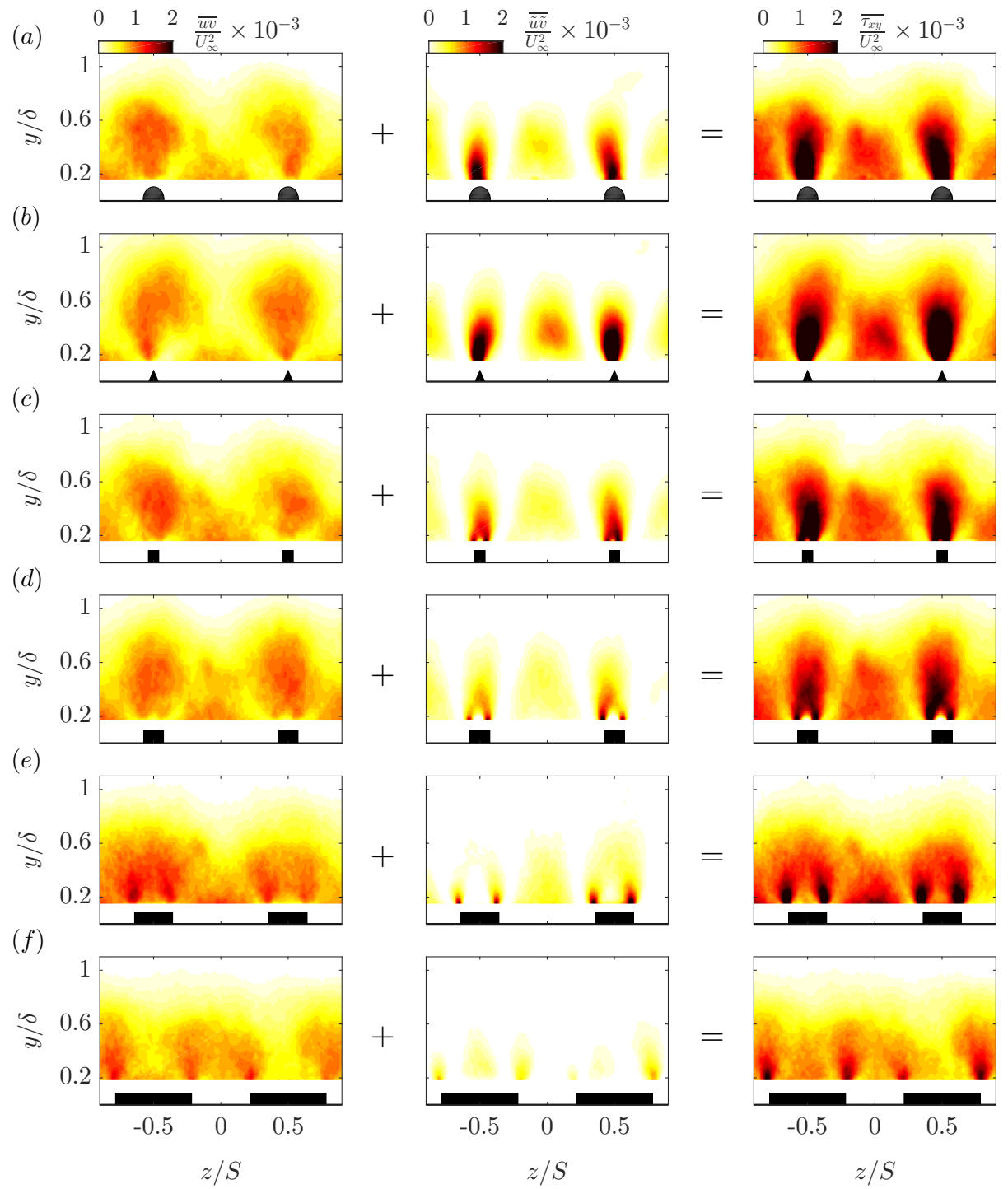

Figure 8: Effect of the different heterogeneous surfaces on the normalised (left panel) turbulent, (middle panel) dispersive and (right panel) total shear-stress. $(a)$ : HS1, $(b)$ : HS2, $(c)$ : HS3, $(d)$ : HS4, $(e)$ : HS5 and $(f)$ : HS6.

and their increase in strength will only result in the rise of magnitude of $\overline{\tau_{x y}}$, without necessarily affecting its spatial distribution. These results are also true for the streamwise stress component which was left out for brevity.

The global behaviour of the dispersive shear-stress is further examined using spanwiseaveraging over a wavelength. This represents a quantification of the overall effect of the flow heterogeneity on the turbulent boundary layer. In figure 9, the contributions of the dispersive shear stresses to total stress are emphasised in figure $9(\mathrm{a})$, plotted in the diagnostic form $\left(\left\langle U / U_{\infty}\right\rangle\right)$. Figure 9 (b) highlights the variation of the peak of $\langle\overline{\tilde{u} \tilde{v}}\rangle /\left\langle\overline{\tau_{x y}}\right\rangle$ with respect to the streamwise circulation $\Gamma^{*}$. Figure $9(\mathrm{c})$ on the other hand illustrates the spanwise-averaged turbulent normal- and shear-stress distributions compared with 
(a)

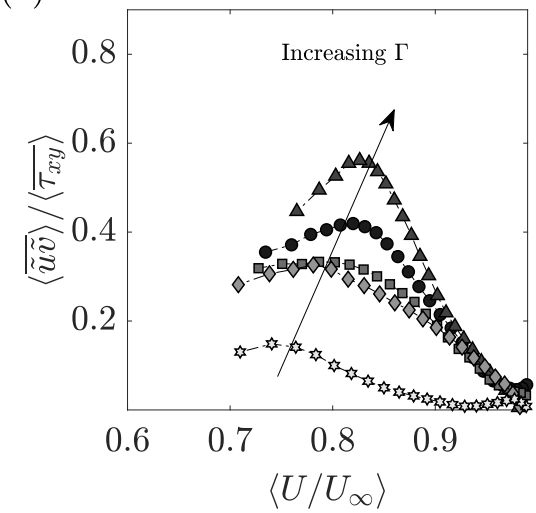

(c)

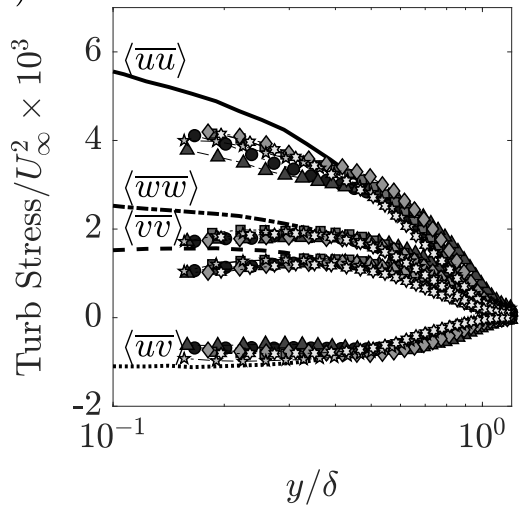

(b)

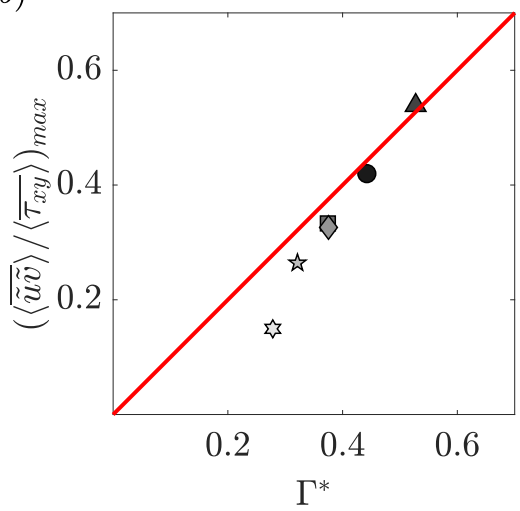

(d)

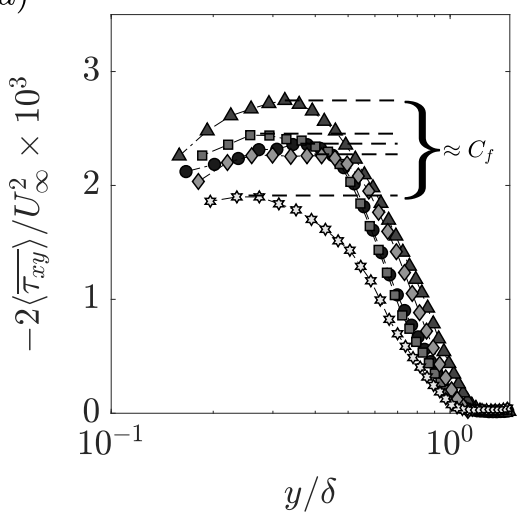

Figure 9: (a) Variation of the dispersive contribution to the total shear-stress in the diagnostic form. (b) Variation of the peak of the dispersive contribution normalised with the total shear-stress with respect to the secondary flow circulation, with the red solid line representing a $45^{\circ}$ slope. (c) Variation of the turbulent shear and normal stresses and comparison with their smooth-wall equivalent profiles, with $\langle\overline{u u}\rangle$ (solid line), $\langle\overline{v v}\rangle$ (dashed line), $\langle\overline{v v}\rangle$ (dashed-dotted line) and $\langle\overline{u v}\rangle$ (dotted line) taken from the smooth turbulent boundary layer data of Kevin et al. (2017) with an $R e_{\tau} \approx 2500$. (d) Wall-normal distribution of the normalised total shear-stress profiles. See table 1 for the symbol legend.

their corresponding smooth wall profiles, while figure $9(\mathrm{~d})$ highlights the wall-normal distribution of the spanwise-averaged total shear-stress defined as

$$
\underbrace{\left\langle\overline{\tau_{x y}}\right\rangle}_{\begin{array}{c}
\text { total } \\
\text { shear } \\
\text { stress }
\end{array}}=\underbrace{\nu \frac{\partial\langle U\rangle}{\partial y}}_{\begin{array}{c}
\text { viscous } \\
\text { shear } \\
\text { stress }
\end{array}}-\underbrace{\langle\overline{u v}\rangle}_{\begin{array}{c}
\text { turbulent } \\
\text { shear } \\
\text { stress }
\end{array}}-\underbrace{\langle\overline{\tilde{u} \tilde{v}}\rangle}_{\begin{array}{c}
\text { dispersive } \\
\text { shear } \\
\text { stress }
\end{array}},
$$

which typically accounts for viscous, turbulent and dispersive shear-stress contributions. As observed from figure 9(a), the dispersive stresses are shown to be significant in magnitude for a large extent of the boundary layer thickness. As opposed to classical homogeneous roughness where it is expected to see their contributions to be only important below the canopy, their effect is presently shown to be substantial even in the outer layer. In fact, these form-induced stresses contribute from $15 \%$ up to $55 \%$ of 
the total shear-stress for the most heterogeneous case. It is additionally shown that at a given distance from the wall $\left(\left\langle U / U_{\infty}\right\rangle=0.9\right.$ or $\left.y=0.6 \delta\right)$, the dispersive stresses are shown to have nearly similar decay rates (specifically the cases HS1-HS4). This can be interpreted as a result of saturation in size of these large-scale secondary motions for a range of $a<\xi<b$, whereby small changes in $\xi$ only enhance or weaken the strength of these secondary motions without necessarily affecting their spatial extent. This further supports the results shown in the figures 6 and 7 for HS1-HS4, which can be considered to be in a "fully" grown heterogeneous regime. As opposed to HS5 and HS6, they were shown to have both weaker and smaller secondary flows, hence their small impact on the primary flow.

Figure 9(a) also underlines that the maximum peak in $\langle\overline{\tilde{u} \tilde{v}}\rangle /\left\langle\overline{\tau_{x y}}\right\rangle$ increases both in magnitude and relative height (expressed by $\left.\left\langle U / U_{\infty}\right\rangle\right)$ when increasing the streamwise circulation, however the peak remains constant when plotted in $y / \delta$. This means that the location of the secondary flows remains unaffected, and the increase in circulation only results in a larger mixing causing a fuller turbulent boundary layer flow. In figure 9 (b), the variation of $\langle\overline{\tilde{u} \tilde{v}}\rangle /\left\langle\overline{\tau_{x y}}\right\rangle_{\max }$ with respect to $\Gamma^{*}$ is emphasised, and is shown to rapidly increase for HS5 and HS6 while almost linearly varies for HS1 to HS4. This result provides further support to the previous interpretation, that the secondary flow formation can undergo different regimes.

The turbulent stresses on the other hand are shown in figure 9(c). The turbulent normal stresses $\langle\overline{u u}\rangle,\langle\overline{v v}\rangle$ and $\langle\overline{w w}\rangle$ as well as the turbulent shear-stress $\langle\overline{u v}\rangle$ seem to have smaller magnitudes in comparison with their equivalent smooth wall profiles, for $y / \delta<0.5$. Moreover, $\langle\overline{u u}\rangle$ and $\langle\overline{u v}\rangle$ in particular are shown to be affected by the degree of the heterogeneity, with the stronger the secondary motions, the weaker these turbulent stresses become, highlighting lack of collapse between cases. Beyond $0.5 \delta$, a weak degree of collapse is observed, however, this is only valid when scaled by $U_{\infty}^{2}$. In fact, when scaled by $U_{\tau}^{2}$ the profiles recover similarity only at the edge of the boundary layer thickness.

Figure $9(\mathrm{~d})$ illustrates the variation of the total shear-stress computed using equation 3.5. The results indicate that overall, the plateau extent and magnitude have been affected by the presence of these large-scale secondary flows. As expected, the magnitude of the plateau increases with increasing $\Gamma^{*}$, which translates to higher wall drag experienced by the surface. Surprisingly, the plateau extent shortens for large $\Gamma^{*}$ and lengthens for smaller $\Gamma^{*}$. This behaviour is caused by the dispersive stress contributions which are amplified by these large-scale secondary flows. In fact, this is more evident when comparing the turbulent shear-stress maps to the dispersive ones as shown in figure 8 . The dispersive stress contributions seem to be accentuated near the core of the secondary flows $(y \approx 0.3 \delta)$, while diminish closer to the wall, leading to the presence of the observed peak in figure $9(\mathrm{a})$. The turbulent stresses on the other hand shown in figure $9(\mathrm{c})$ seem to have a nearly constant contribution up to $50 \%$ of the boundary layer thickness when spanwise-averaged. Therefore, the presence of a peak instead of a classical plateau in $\left\langle\overline{\tau_{x y}}\right\rangle$ is considered to be a consequence of the increased lateral motions which enhance the secondary flows and lead to higher momentum transport. These results are also in agreement with the recent studies that assessed the contributions of the dispersive stresses (Chan et al. 2018; Vanderwel et al. 2019; Meyers et al. 2019). They found that these forminduced stresses are prominent within the canopy but can remarkably increase spatially depending of the surface heterogeneity length scale. For a certain range of spanwise wavelengths, the turbulent stresses are shown to exhibit strong spanwise gradients which lead to the added dispersive stresses that can extend up two thirds of the flow.

The impact of the secondary flows on the outer-layer similarity is examined in figure 10. Figure 10(a) shows the spanwise-averaged mean velocity profiles in defect form scaled 
(a)

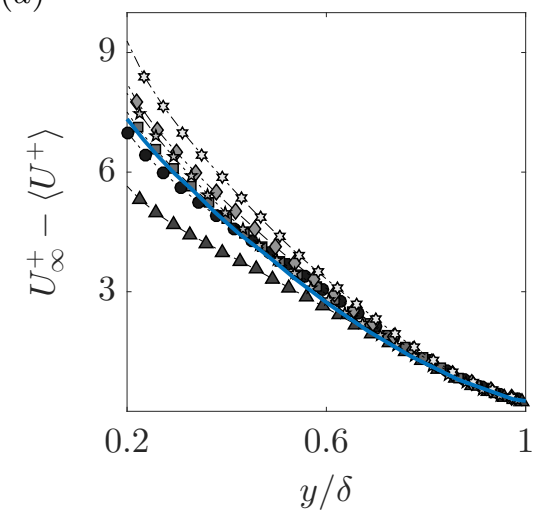

(b)

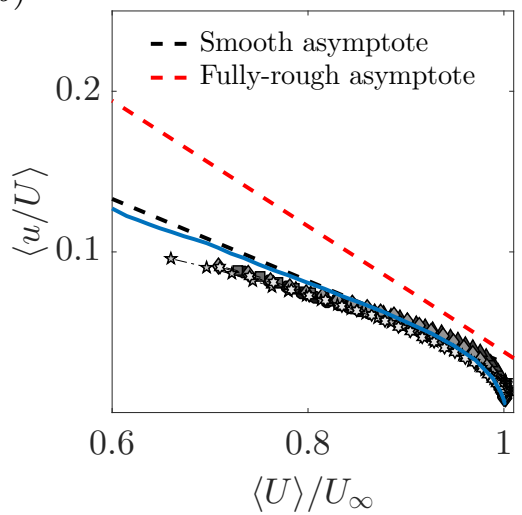

Figure 10: Effect of the different heterogeneous surfaces on $(a)$ spanwise-averaged mean velocity profiles in the defect form normalised with the friction velocity determined from the total shear-stress. (b) Outer scaling of spanwise-averaged streamwise turbulence intensity in the diagnostic-plot form. The blue solid-line represents the smooth-wall data from Medjnoun et al. (2018). See table 1 for the symbol legend.

with the friction velocity taken from the total shear-stress plateau. On the other hand, figure 10(b) highlights the spanwise-averaged streamwise turbulence intensity profiles plotted in the diagnostic form introduced by Alfredsson et al. (2011). There is marginal lack of collapse in the velocity deficit for all the different surfaces when compared to the smooth wall data. The absence of collapse extends up to $y=0.6 \delta$, whose recovery seems to occur when the dispersive stresses decay faster as shown in figure 8 . The turbulence intensity profiles are even more affected by the presence of these large secondary motions, with a recovery only occurring at the edge of the boundary layer (not shown here for brevity). The above observations are consistent with previous results both from the ridge-type and the roughness-type studies by Chan et al. (2018) and Chung et al. (2018) respectively. They essentially showed for spanwise wavelengths comparable to the dominant length scale of the flow, the secondary flows occupy a large portion of the primary flow, leading to strong dispersive stresses (extended roughness sublayer) which results in the breakdown of both global and local outer-layer similarity.

A good collapse is observed for the variance profiles as shown in figure 10(b), not only between the cases, but also with the smooth wall. This suggest that regardless of the changes in the secondary flow circulation, the streamwise turbulence intensity along with the mean flow change proportionally in the outer region. Moreover, the collapse of the profiles with the smooth wall irrespective of a non-zero roughness function $\Delta U^{+}$ (representative of the drag changes shown in figure 3) does not seem to be captured in the diagnostic plot. This observation is somewhat inconsistent with the conclusions of Castro et al. (2013) who demonstrated that increasing $\Delta U^{+}$leads to a higher slope in $u / U$ till reaching the fully-rough trend (represented by the red-dashed line in figure 10(b)). One possible reason for the current behaviour is that the value of the roughness function is small $\left(\Delta U^{+} \leq 4\right)$. Additionally as discussed in $\S 3.1$, the absence of form drag contribution to $\Delta U^{+}$due to lack of streamwise rough protrusions can inhibit the increase of the streamwise turbulence level relative to the mean flow. Similar results are also reported in our previous study regarding local profiles across the spanwise direction (Medjnoun et al. 2018). 


\subsection{Effect on the coherent structures in the outer region}

To further investigate the influence of the surface heterogeneity on the characteristics of the turbulence structures, the two-point correlation function of the velocity fluctuations is examined. This allows qualitative and quantitative characterisation of the spatial structures at different critical locations such as the high- and low- momentum pathways that characterise this flow (Wu \& Christensen 2010; Yang \& Anderson 2017; Bai et al. 2018; Kevin et al. 2019). The two-point correlation coefficient tensor $R_{u_{i} u_{j}}$ in the $(y, z)$ plane is defined as,

$$
R_{u_{i} u_{j}}\left(y_{r e f}, z_{r e f}\right)=\frac{\overline{u_{i}\left(y_{r e f}, z_{r e f}\right) u_{j}(y, z)}}{\sigma_{u_{i}}\left(y_{r e f}, z_{r e f}\right) \sigma_{u_{j}}(y, z)},
$$

where $y_{r e f}$ and $z_{\text {ref }}$ are the reference wall-normal and spanwise locations at which the correlation is examined. $\sigma_{u_{i}}\left(y_{r e f}, z_{r e f}\right)$ is the standard deviation of $u_{i}$ at $y_{r e f}$ and $z_{r e f}$ and $\sigma_{u_{j}}(y, z)$ is the standard deviation of $u_{j}$ in the $(y, z)$-plane. Figure 11 illustrates the two-dimensional autocorrelation maps of the streamwise velocity fluctuation $R_{u u}$ at $y / \delta=0.4$ determined at three different spanwise reference locations $(z / S=-0.5,-0.25$ and 0 from left to right panels) for four different heterogeneous surfaces (HS1, HS2, HS4 and HS6). The wall-normal location was chosen to show representative existence or lack of structural similarity in the outer layer while the spanwise locations are chosen to investigate structural changes in the spanwise direction.

Significant changes in the spatial coherence in both the wall-normal and spanwise directions are observed for the different locations. The correlations maps indicate that at the LMPs (left panel of figure 11), the structures are skewed in the wall-normal direction, but symmetric in the spanwise direction. They also highlight the emergence of negatively correlated regions shown as the black dashed contour flanking the either sides of the ridges. Their spanwise extent appears to extend away from the wall while they shrink when closer to the ridge. In contrast, the structures observed at the HMPs show an opposite behaviour with a much larger spatial coherence near the wall (right panel of figure 11) as opposed to a narrower correlation away from the wall. Two negatively correlated regions exist in the spanwise direction with a larger distance away from their main lobe compared to the LMP structure. The asymmetric nature in the horizontal plane of these structures seems to link with the presence of the large-scale secondary motions. In fact, if we pick a point near the wall $(y=0.2 \delta)$, strong inward radial motions are expected above the ridge whereas outward radial motions occur at the valley. Likewise, at a location away from the wall $(y=0.6 \delta)$, the opposite behaviour is expected to occur. Hence the non-symmetrical aspect of the coherent structures observed above the peaks and valleys.

The correlation maps in the mid-plane between HMPs and LMPs (middle panel in figure 11) indicates that both their spatial extent as well as their orientations are affected. A significant increase in the coherence of the structures accompanied with a substantial tilt is shown through the black contour level, along with the rise of two nonsymmetrical negatively correlation regions. This inclination is believed to be caused by the strong radial motions induced by the presence of these large-scale secondary flows, which result in the spanwise mean shear flow. In fact, their inclination angles seem to line up reasonably well with the normal to the isolines of the streamwise velocity map (as shown in the figure 4). This is more evident with the observation made for the HS6 case which showed a reversal in the direction of the tilt similar to the swap in the LMP and HMP locations reported for the same surface. Qualitative similarities across cases are observed when comparing the extent of the coherent structure at the peaks and 
(a)

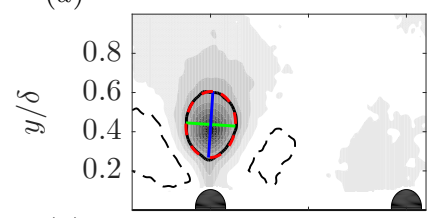

$(d)$

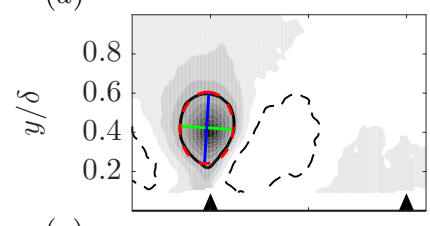

$(g)$

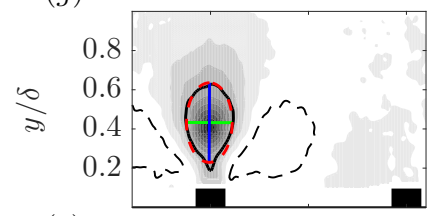

$(j)$

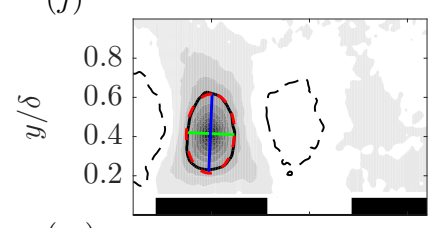

$(m)$

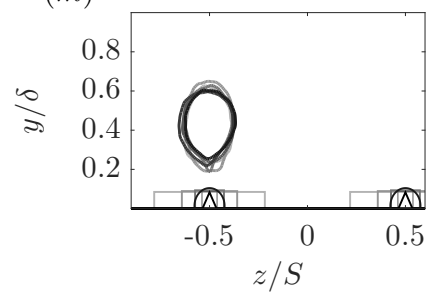

(b)

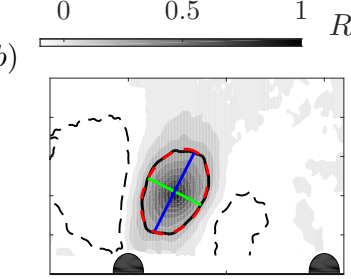

(e)

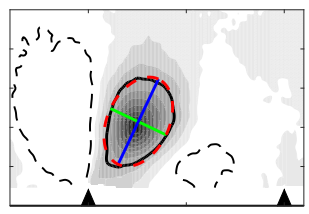

$(h)$

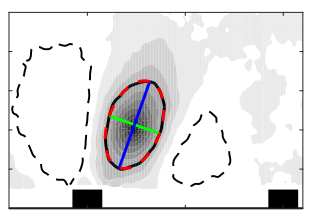

(k)

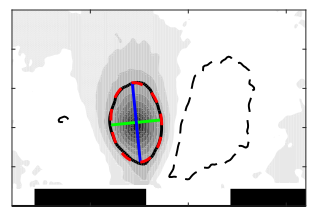

$(n)$

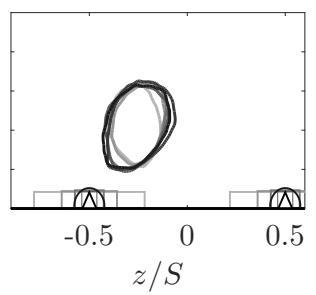

(c)

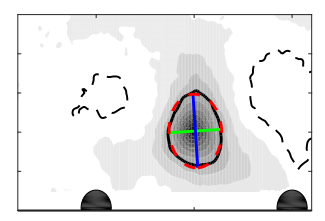

(f)

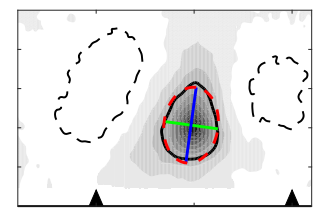

(i)

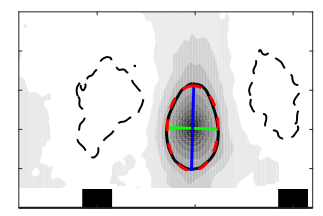

(l)

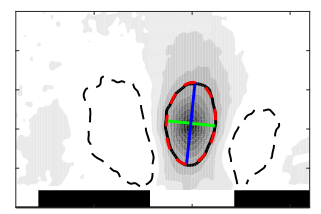

$(o)$

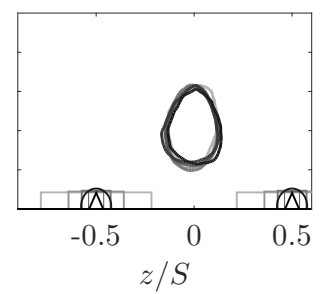

Figure 11: Spatial autocorrelation maps of the fluctuating streamwise velocity $R_{u u}$ in the cross-plane at $y_{\text {ref }}=0.4 \delta$ over three spanwise locations: (left panel) peak, (middle panel) mid-peak-valley distance and (right panel) valley. $(a-c)$ : HS1, $(d-f)$ : HS2, $(g-h)$ : HS4, $(j-l)$ : HS6 and $(m-o)$ : a selected contour level of all the cases superimposed. The black contour level represent $R_{u u}=0.3$. The corresponding ellipsoid fit is represented in red dashed contour with its major and minor axes in blue and green lines respectively. The dashed black contour level represents $R_{u u}=-0.1$.

valleys as shown in figure $11(\mathrm{~m})$ and (o). The exception remains the HS6 case, where the turbulence structure seems to have been altered due to the swap of the LMP and HMP locations.

Quantitative changes in the turbulence structures (coherence inclination angles and length scales) are computed from the $R_{u u}$ map in the $(y, z)$-plane. This is done by fitting an ellipsoid to a given contour level (shown with a black solid-curve in figure 11 at $R_{u u}=$ 0.3 ). The parameters of this fitted ellipse (red dashed-curve) include the two orthogonal axes with a major axis (shown in blue) and a minor axis (shown in green) represent characteristic length scales in the wall-normal and spanwise directions. An overall length 

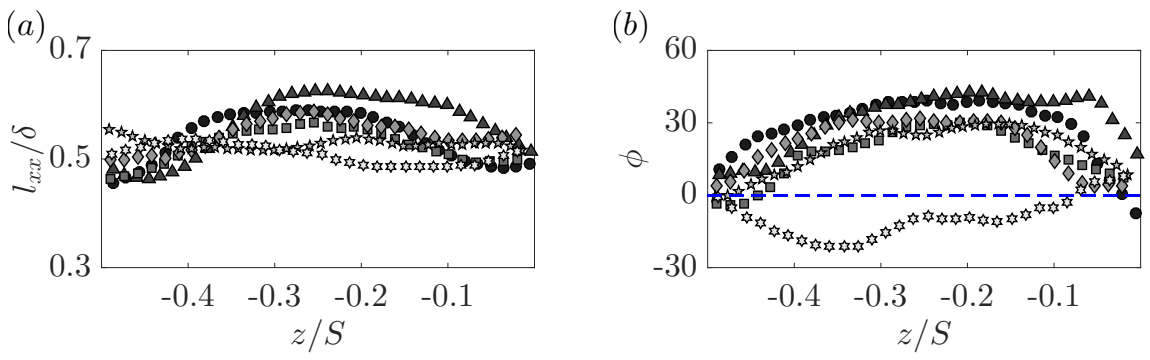

Figure 12: Spanwise variation of the $(a)$ normalised autocorrelation length scale determined at $R_{u u}=0.3$ and $(b)$ the corresponding structure inclination subjected to different surface heterogeneities. Positive and negative angles above and below the blue dashed line referred to clockwise and anti-clockwise inclination of the structure. See table 1 for the symbol legend.

$l_{x x}$ shown in figure $12(\mathrm{a})$ is estimated by taking the square root of the sum of the squares of the minor and major axes of the ellipse, while the inclination angle $\Phi$ is just the major axis clockwise rotation from the wall-normal direction as shown in figure 12(b). Both these values seems to have been affected by the surface heterogeneities. The length scale $l_{x x}$ seems to grow between the high- and low-momentum pathways especially where the spanwise mean shear flow is the highest. This is accompanied by a modification in the turbulence structure shapes which show elongated oblique coherence characterised by their inclination.

These observations are also in agreement with other recent studies over the striptype surfaces from Bai et al. (2018) and Kevin et al. (2019). The results from the wallparallel Stereo-PIV performed in the log-region by Bai et al. (2018) revealed that near the surface discontinuity, the coherent structures presented a yawed coherence due to the lateral inflow. This region which is home to strong wall-normal vortices induces the high and low speed streaks to be arranged above the high roughness (HMPs) and low roughness (LMPs) respectively. This is furthermore supported by the study of Kevin et al. (2019) who investigated the characteristics of these coherent structures in multiple streamwise, spanwise as well as wall-parallel planes in the converging-diverging riblet roughness. They found that between the HMPs and LMPs, the region is home to an appearing "detached" coherent structure which is characterised by a tilt in the wallnormal and streamwise direction that enhances lateral momentum transfer. This is a further evidence that structural similarity has been altered by the secondary flows.

\subsection{Effect on the wake intermittency}

The turbulent/non-turbulent interface (TNTI) has been widely explored in many flows, such as in wakes, jets and turbulent boundary layers (Bisset et al. 2002; Westerweel et al. 2005; Laskari et al. 2018). The TNTI represents an instantaneous thin layer that isolates regions of turbulent motions from regions of the non-turbulent flow (Corrsin \& Kistler 1955). The interface is characterised by a simultaneous occurrence of small and largescale processes termed as "nibbling" and "engulfment", representative of viscous and inertial mechanisms, inducing turbulence-free fluid entrainment towards the turbulent flow (Chauhan et al. 2014). In the presence of surface spanwise heterogeneity, both the inner and outer regions were shown to be affected by the large-scale secondary motions, which rearrange the structural organisation of the turbulence (Yang \& Anderson 2017; 


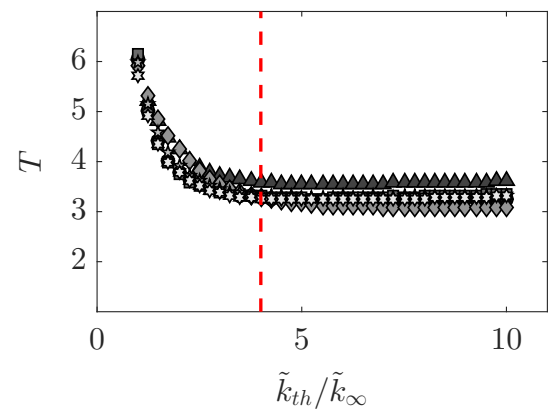

Figure 13: Effect of different threshold levels on the tortuosity parameter for the different heterogeneous surfaces. The vertical red dashed-line represents the selected threshold $\tilde{k}_{t h} / \tilde{k}_{\infty}=4$ for the TNTI analysis. See table 1 for the symbol legend.

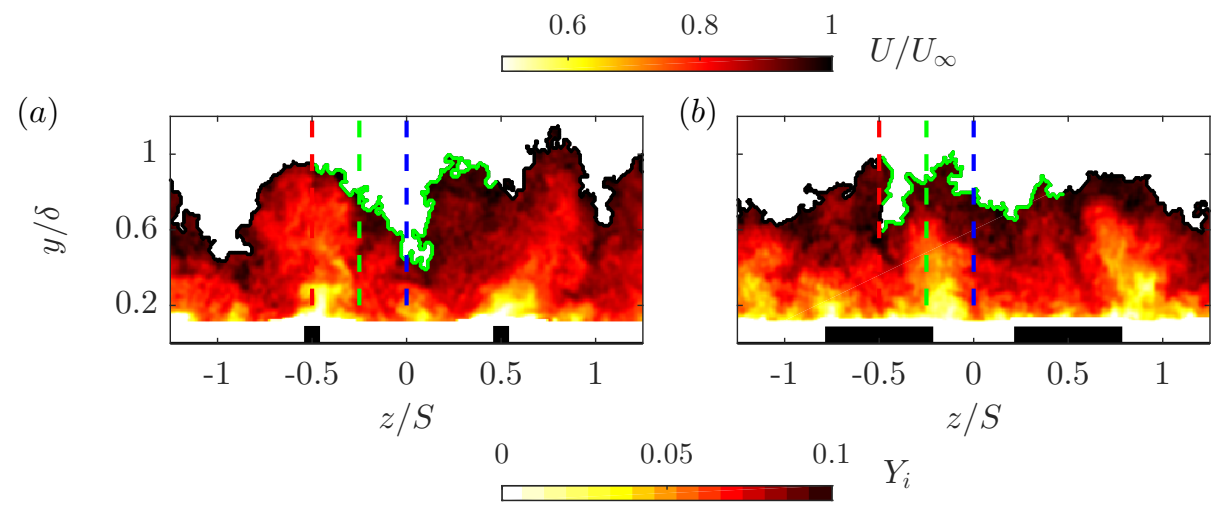

(c)

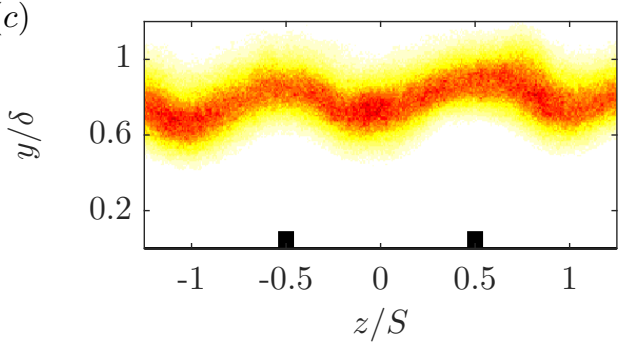

$(d)$

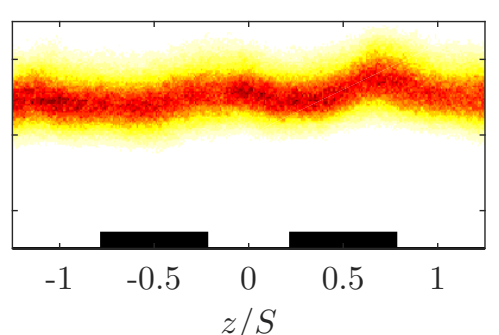

Figure 14: Example of the application of the turbulent kinetic energy deficit criterion to an instantaneous streamwise velocity map for (a) HS3 and (b) HS6. The turbulent/nonturbulent interface outline is highlighted with a black line. The arc-length used to determined the tortuosity in figure 13 is shown inside one wavelength in green. The colourcoded vertical dashed lines are the spanwise locations over which the local intermittency profiles are evaluated as shown in figure $15(a)$ and $(b) .(c)$ and $(d)$ represent the ensemble averages of the spatial occurrence on the TNTI in the $(y, z)$-plane for HS3 and HS6 respectively.

Kevin et al. 2017; Bai et al. 2018; Kevin et al. 2019). Therefore, this rises the interrogation on how does the wake intermittency re-adapt to the structural changes observed before.

The interface detection method currently employed is similar to that defined by Chauhan et al. (2014), based on a local turbulent kinetic energy deficit detector $\tilde{k}_{t h}$, of a given threshold that separates the instantaneous flow into a turbulent and non-turbulent 
flow. However, the threshold selection is shown to be specific to each experiment and can be influenced by the turbulence level in the free stream, the measurement systematic and statistical uncertainties. Consequently, in order to have a consistent way of defining suitable thresholds for the different cases, a variable $T$ defined as the "tortuosity" is determined for different levels of thresholds. The tortuosity essentially represents the arc-length of the interface $l_{i}$ within a given unit distance, herein defined as $T=l_{i} / S$. Figure 13 illustrates the variation of the tortuosity as a function of the threshold level $\tilde{k}_{t h}$ normalised by the turbulent kinetic energy deficit in the free stream $\tilde{k}_{\infty}$. The results indicate that beyond a certain level, the tortuosity becomes constant irrespective of changes in the threshold level $\tilde{k}_{t h} / \tilde{k}_{\infty}$. The value $\tilde{k}_{t h} / \tilde{k}_{\infty}=4$ was found to correspond to a reasonable threshold level that yields a consistent comparison between cases. In this case, $4 \tilde{k}_{\infty}$ ranges between $0.12-0.25$, which are between a factor one and two of the value used by Chauhan et al. (2014). However, as mentioned above, these values are not expected to remain constant from one experiment to another.

To demonstrate the effect of the surface heterogeneity on the interface, two examples for HS3 and HS6 are highlighted in figures 14(a) and (b) respectively. The TNTI is shown in figures 14(a) and (b) as the black contour delineating two distinct regions in the instantaneous streamwise velocity map; turbulence-free stream above and turbulent regions below the interface outline. The arc-length of the TNTI within a spanwise unit distance used to determine the tortuosity is highlighted in green. Figure 14(a) clearly indicates that substantial distortions are present at the interface outline, with alternating large-scale bulges and valleys above the elevated and recessed surfaces, respectively. This observation remains consistent for all the cases except HS6 as shown in figure 14(b), which showed the occurrence of the opposite behaviour.

In order to get a quantitative assessment of these differences, the TNTIs of all the snapshots are superimposed to obtain an ensemble average as illustrated in figures 14(c) and (d). As expected, the wall-normal location of the TNTI appears to undergo a similar periodicity as the mean flow in the spanwise direction, with alternating high and low TNTI locations above the elevated and recessed surface respectively. Furthermore, the changes observed in the mean and the turbulent characteristics for HS6 are also reflected in figure $14(\mathrm{~d})$, which is characterised by a swapping of the high and low TNTI locations. The spanwise variation of the interface seems to also have a subtle dependence on the strength of secondary flows. In fact, the maps of the TNTI height $Y_{i}$ indicate that the peak-to-peak for HS3 is higher than that of HS6. However, this is not completely clear when the tortuosity of the interface is examined as shown in figure 13. It appears that the most tortuous interface is HS2, which was shown to be the most heterogeneous flow. Nevertheless, the rest of the cases seem to have relatively similar values of $T$ which range between 3.2 and 3.6 for the different cases.

It is perhaps also interesting to point out that the values of $T$ are in a reasonable agreement with the predicted value by Chauhan et al. (2014) for a smooth wall turbulent boundary layer. They hypothesised that based on the fractal nature of the TNTI, its length in the spanwise direction is expected to be three times the distance where it sits on, similar to that in streamwise direction. In the present study, the induced crossplane secondary motions caused by the surface heterogeneity have effectively altered the interface, hence the slightly larger values of the tortuosity. Similar findings have also been recently reported by Kevin et al. (2017), who observed strong spanwise waviness of the TNTI. Their analysis was based on the identification of "pockets" of turbulence-free fluid within the turbulent flow below the interface outline. After looking at both the spatial distribution of these non-turbulent pockets as well as their frequency of occurrence, they observed that their manifestation is two times more probable over the downwash than 

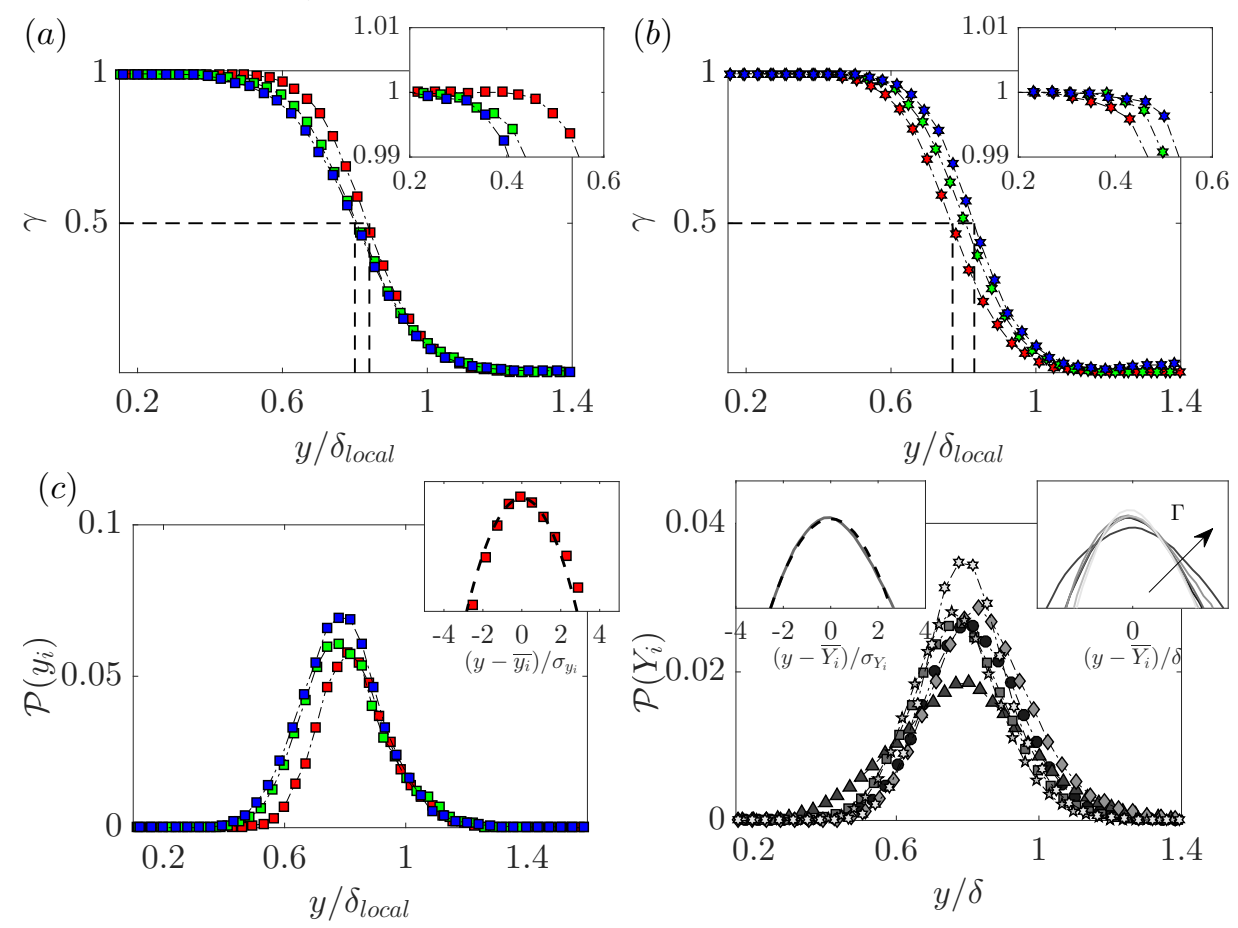

Figure 15: Wall-normal distribution of the local intermittency profiles $\gamma$ for $(a)$ HS3 and (b) HS6 at $z / S=-0.5,-0.25$ and 0 for the red, green and blue markers respectively. (c) The corresponding probability density function $\mathcal{P}\left(y_{i}\right)$ for HS3 with the inset comparing the profile at $z / S=-0.5$ (red squares) to a Gaussian distribution (dashed line) normalised with their variance. $(d)$ The global probability density function of the TNTI $\mathcal{P}\left(Y_{i}\right)$ locations for the different cases. The left and right insets of $(d)$ highlight the profile of HS3 (solid line) compared with a Gaussian distribution (dashed line) normalised by their variance, and the profiles of all the cases colour-coded as shown in table 1's symbol legend.

the upwash regions. They showed that this was due to large-scale free stream engulfment occurring close to the wall, in contrast to less recurrent pockets over the upwash regions which appeared far from the wall.

The intermittency $\gamma$ indicates the proportion of time a flow is turbulent at a given point in the $(y, z)$-plane. Consequently, $\gamma$ tends to 1 near the wall whereas tends to zero near the free stream. The local intermittency profiles are shown for three different spanwise locations for HS3 and HS6 in figures 15(a) and (b) respectively. The different colours highlight the three spanwise locations illustrated with vertical coloured dashedlines in figures 14(a) and (b). The profiles indicate the intermittency to have a constant upper bound beyond which the flow remains statistically laminar, when the wall-normal distance is scaled with the local boundary layer thickness. On the other hand, the insets of figures 15(a) and (b) reveal that the lower bound changes according to the spanwise location. For example, figure 15(a) indicates that the intermittency extent is wider at the valley $(z / S=0$ and -0.25$)$ than over the ridge $(z / S=-0.5)$ which correspond to the downwash and upwash regions respectively. This result is in fact not surprising since the ridges give rise to high-turbulent fluxes, which are then advected vertically, causing the reduction in the wake intermittent activity at the ridge. Conversely, the recessed 
regions are dominated by downwash motions transporting low-turbulent fluxes towards the near wall rendering the intermittent region larger. As expected, these results are shown to be valid for all the cases with the exception of HS6. This is demonstrated in figure 15(b) which highlights the occurrence of an opposite behaviour, where the profiles above the ridge appear to have an extended intermittent region, although the differences being weaker. These changes between HS6 and HS3 (as well as the rest of the cases) are expected to take place since we previously observed the HMPs and LMPs to have swapped locations. This wake behaviour means that the HMPs (downwash) will always induce larger intermittency extents, whiles the LMPs (upwash) will generate shorter intermittency extents.

Finally, the probability density function of the local TNTIs, $\mathcal{P}\left(y_{i}\right)$, is examined in figure 15(c) for HS3. The profiles reveal that the local mean heights of the TNTI $\overline{y_{i}}$ vary marginally in the spanwise direction. This is also highlighted by the vertical and horizontal black dashed-lines which correspond to $\gamma=0.5$ in figures $15(\mathrm{a})$ and (b). The structural modifications in the outer region also appear to have affected the Gaussian nature of the TNTIs, specifically at the LMP. This is illustrated in the inset of figure 15(c) which compares the local profile at the upwash region (red squares) against a Gaussian distribution (black dashed-line), which showed a degree of skewness towards to free stream. This observation, however, is only true in the local sense. Globally, the profiles are shown to remain Gaussian regardless of the surface heterogeneity. This is depicted in figure 15(d) which inspects the global probability density function $\mathcal{P}\left(Y_{i}\right)$ across cases. Despite the differences in the mean TNTI height $\overline{Y_{i}}$ which ranges between $0.77 \leq \bar{Y}_{i} / \delta \leq 0.86$, the distributions appear to have a good degree of symmetry. As a matter of fact, $\mathcal{P}\left(Y_{i}\right)$ for HS3 is presented in the left inset of figure $15(\mathrm{~d})$, and shows an excellent degree of collapse with the Gaussian distribution (black dashed-line). This indicates, in spite of the presence of large-scale secondary flows, the Gaussian nature of the TNTI globally remains unaffected. The secondary flows are shown to mainly induce changes in the extent of the intermittent regions. The right inset of figure $15(\mathrm{~d})$ displays that the TNTI extent becomes larger for cases that exhibit stronger secondary motions. This is shown by the changes in the standard deviation of the interface which ranges between $0.11 \leq \sigma_{Y_{i}} / \delta \leq 0.19$ from the least to most heterogeneous case.

Overall, the results highlighted from the TNTI analysis are consistent with the recently observed changes in the wake strength parameter $\Pi$ reported by Medjnoun et al. (2018). In fact, by measuring directly the local friction velocity and the corresponding velocity profile across different spanwise locations, they showed the wake strength parameter to be affected by the surface spanwise heterogeneity (hence the flow heterogeneity). They found that the elevated and recessed regions of the surface (corresponding to the LMPs and HMPs respectively) resulted in low and high values of $\Pi$ respectively. As indicated by Krug et al. (2017), the wake strength parameter stems from a dual effect of the velocity jump across the TNTI as well as the uniform momentum zones. Therefore, the previously observed changes in the wake parameter are probably due to the changes in the intermittent activity across the span. While the current results highlight the effect of the large-scale secondary motions on the wake intermittency, further studies remain necessary to also examine their impact on the uniform momentum zones.

\section{Discussion}

Two questions that drew the attention of researchers have been the prediction of the high- and low-momentum pathways for various wall-conditions and the identification of important surface features that can scale the secondary flows. Based on existing 
(a)
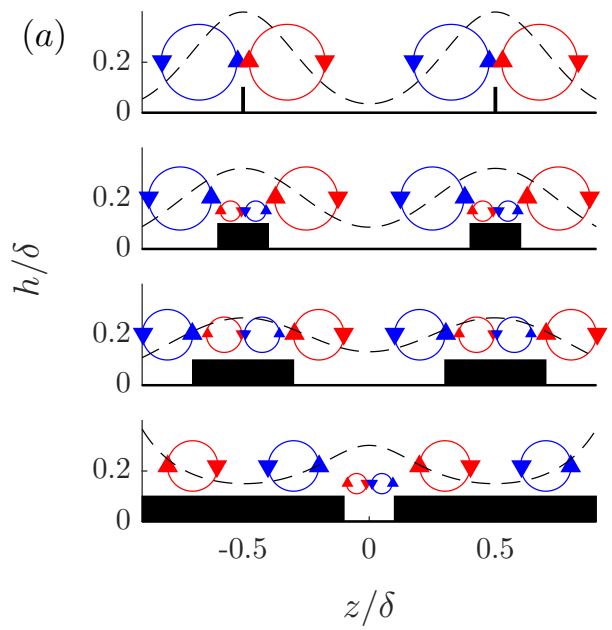

(b)
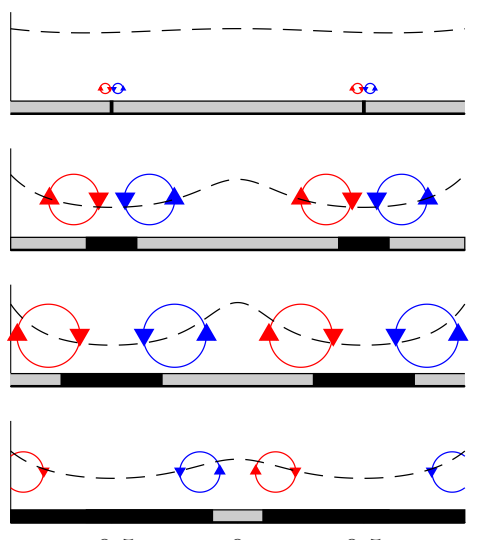

$-0.5$

0

0.5

$z / \delta$

Figure 16: Sketch illustrating the effect of the ridge/roughness width for the $(a)$ ridgetype and $(b)$ strip-type heterogeneity. The secondary motions and mean flow curvature are highlighted in clockwise/anticlockwise red/blue circles and black dashed lines.

literature, we found two main categories of surfaces that seemingly exhibited similar flow attributes, yet have different triggering mechanisms of secondary flows.

For the skin friction based heterogeneity whose surface alternates between high and low wall shear-stress (due to surface roughness or superhydrophobic surfaces etc), both the width of the high/low skin friction and the characteristic spanwise wavelength were shown to be relevant. Willingham et al. (2014) showed that by keeping the spanwise wavelength constant, increasing the high drag width produced stronger mean flow heterogeneity. They showed that the HMPs and LMPs systematically occurred above the high and low wall shear-stress, respectively. On the other hand, Stroh et al. (2016) varied both the spanwise wavelength and the low shear-stress width and reported their important influence on the flow topology. They showed that for a relatively thin strip of the high shear-stress, HMPs and LMPs occurred above the low and high wall shear-stress, respectively. For increased strip widths, the opposite behaviour occurred. Chung et al. (2018) have also showed by keeping the high-to-low roughness widths constant and just increasing the spanwise wavelength, the HMPs and LMPs are always observed above the high and low wall shear-stress, respectively. The exception being that for very large wavelengths (both large high/low skin-friction widths), the HMPs and LMPs appeared above the low and high skin friction, respectively. This is a confirmation that local homogeneity of the flow can be recovered (away from the discontinuity), with high and low roughness producing thicker and thinner boundary layer thickness. Hence, the different studies indicate that perhaps more than one length scale should be considered for characterising the surface heterogeneity.

Most of the previous studies for the ridge-type heterogeneous surfaces showed that the spanwise wavelength is relevant for the presence of the secondary flows (Nezu \& Nakagawa 1984; Vanderwel \& Ganapathisubramani 2015; Yang \& Anderson 2017; Hwang \& Lee 2018). Analogous to the study of Willingham et al. (2014), the current results highlighted the importance of an additional surface feature depicted by the ratio of the recessed and elevated regions in addition to the spanwise wavelength. This analogy between the two wall-conditions is sketched in figure 16 (a) and (b) for the ridge- and roughness-type heterogeneity, respectively, for a fixed wavelength $S$. By drawing on the results from 
the current and previous studies, we can identify notable differences in the two types. Considering a generic shape such as a rectangle of a given height and varying widths to represent the ridge-type heterogeneity, we can observe manifestations of secondary motions in the form of large-scale streamwise rollers flanking the ridges, and perhaps tertiary structures as shown in this and many other studies. For very thin ridges, strong secondary motions are expected (similar to HS2) with upwash and downwash occurring above and in-between the ridges. Increasing the width results in the appearance of the tertiary structures competing with the large secondary structures above the ridge, thereby weakening the mean flow heterogeneity. Increasing the width beyond a certain limit results in the swap of HMPs and LMPs, albeit with a lower level of the flow heterogeneity, till the flow reaches homogeneity again. In the case of roughness-type heterogeneity, the secondary flows are expected to remain confined in the vicinity of the stress discontinuity for the limits of very wide/narrow strips. This results in a weak Reynolds shear-stress heterogeneity causing the base flow to remain homogeneous. Between these two limits, the secondary flows reach their full potential over a range of widths, then will decrease eventually when increasing/decreasing above/below a certain limit.

In view of these results, it is clear that the spanwise wavelength is not sufficient on its own to describe the flow heterogeneity. A surface heterogeneity parameter $\mathcal{H}$ can be considered as a combination of $S$ and an additional surface parameter $\xi$ :

$$
\mathcal{H}=F\left(\frac{S}{\delta}, \xi\right)
$$

In case of a ridge-type heterogeneity, we have shown in this study that $\xi$ can be expressed as a ratio of the area/perimeter of the elevated and recessed regions. This was shown to capture both the shape and width variations of the ridges. In the case of a roughness-type, $\xi$ can relate to a ratio of the high-to-low roughness (or high-to-low shear-stress) weighted by their respective widths. Overall, the spanwise heterogeneity can be thought of as a combination of at least two parameters. First, a spanwise characteristic wavelength $(S / \delta)$, which represents an onset condition necessary to trigger large scale spanwise fluctuations. Second, a geometrical parameter (represented herein as the ratio of the recessed and elevated areas $(\xi)$ necessary to amplify or reduce these radial motions. Therefore, one can influence the secondary motions by varying $S / \delta$ while keeping $\xi$ constant (see e.g. Chan et al. (2018)), alternatively by keeping $S / \delta$ constant and changing $\xi$ (such as in the present investigation). For the ridge-type surfaces considered here, the relationship between the secondary flow circulation and $\xi$ appears to be linear for a fixed $S / \delta$ over the range of $\xi$ examined here.

The second question that has garnered attention is the spanwise locations of the HMPs and LMPs for the ridge-type heterogeneity. Several studies reported similar findings to ours when $S / \delta \approx \mathcal{O}(1)$ (Nezu \& Nakagawa 1984; Colombini 1993; Wang \& Cheng 2006; Vanderwel \& Ganapathisubramani 2015; Hwang \& Lee 2018; Vanderwel et al. 2019). Contrastingly, other recent investigations showed that the opposite scenario can occur (Mejia-Alvarez \& Christensen 2013; Barros \& Christensen 2014; Yang \& Anderson 2017; Awasthi \& Anderson 2018). However, one of the key differences between these two sets of studies is the presence/absence of streamwise homogeneity of the surface. In fact, all the studies that reported the upwash above the surface elevations used streamwise continuous ridges, in contrast with the studies that reported downwash to occur above the elevated regions. The highly irregular roughness of Mejia-Alvarez \& Christensen (2013); Barros \& Christensen (2014) and the highly ordered roughness of Yang \& Anderson (2017); Awasthi \& Anderson (2018) clearly exhibit wake producing protrusions, which 
induce form-drag contributions. Therefore, despite these surfaces being topographically heterogeneous, they appear to act more like high/low wall shear-stress producing surfaces, in contrast with the previous ones which are predominantly viscous drag producing surfaces. The exception was the surface studied by Vanderwel \& Ganapathisubramani (2015) which also contained form-drag producing pins. However these protrusions were homogeneously distributed over the surface, meaning that their overall contributions remained uniform as opposed to Yang \& Anderson (2017) and Awasthi \& Anderson (2018) which were localised only at the surface elevations. Although this explanation may seem plausible following the rule of "high" and "low" roughness generating downwash and upwash respectively, this is clearly not the case for the staggered sinusoidal roughness investigated by Chan et al. (2018). In fact, they reported the HMPs and LMPs to be located between the peaks and valleys of the topography, corresponding to regions of low and high wall shear-stress. Consequently, the differences between the driving mechanisms behind the direction of the secondary flows for staggered and non-staggered roughness needs further exploration.

Finally, the utility of outer-layer similarity in flows with secondary motions has been questioned and examined. Based on the experimental work of $\mathrm{Wu} \&$ Christensen (2007, 2010) and Pathikonda \& Christensen (2017), both mean flow and structural similarity were shown to exist despite the emergence of secondary flows. This is in contrast to other investigations that showed outer-layer similarity to be affected in first and second order moments as well as its spectra (Yang \& Anderson 2017; Medjnoun et al. 2018; Chung et al. 2018). Chan et al. (2018) provided useful insight onto the conditions of validity of the similarity hypothesis, based on the quantification of the dispersive stresses. They showed that as long as these form-induced stresses remained only prominent near the canopy layer, the turbulent structures in the outer region remain unaffected by the secondary flows. However this only occurs for very small spanwise wavelengths. As soon as the wavelength is comparable to the dominant length scale of the flow, the secondary motions occupy a large portion of the flow, which induce dispersive stresses that extend farther in the outer region. Our current findings also corroborate these observations such that the cases presenting strong secondary motions, showed lack of outer-layer similarity. It is also important to recall that the primary condition for outer-layer similarity is a large scale separation (Jiménez 2004; Castro 2007; Flack \& Schultz 2014; Squire et al. 2016). In the case of the study by Barros \& Christensen (2014), their surface roughness exhibited a wide range of multiscale features as opposed to the other studies. These roughness features are likely to enhance near wall mixing, increase the overall drag and the scale separation. This combined with perhaps relatively mild dispersive stresses can result in the validity of outer-layer similarity.

\section{Conclusions}

A turbulent boundary layer flow over smooth heterogeneous surfaces has been experimentally studied. We specifically looked at the influence of the different surface heterogeneities on the secondary flows that developed under these conditions. Oil-film interferometry along with Stereo-PIV measurements allowed us to evaluate the impact of these heterogeneous surfaces on the skin friction, the mean and turbulent flow properties.

In the presence of ridge-type surface heterogeneity, the spanwise distribution of the skin friction varies as a consequence of the mean flow heterogeneity. As a result, strong spanwise skin-friction gradients are observed, and are located near the regions of high spanwise mean shear. The average frictional drag was directly assessed and was shown to be both a function of Reynolds number as well as the surface heterogeneity. It is 
speculated that these surfaces do not allow the flow to transition to a "fully" rough regime, as the roughness function is entirely driven by the viscous drag and has no form-drag due to lack of streamwise roughness protrusion.

The mean vertical velocity component emphasised the importance of both the ridge shape and width. It is shown that beyond a given value of width, a swap between the HMPs and LMPs can occur. For $(S-W) / \delta>0.5$, the HMPs and LMPs were consistently shown to occur above the recessed and elevated regions of the surface. The modifications were also evident in the streamwise vorticity highlighted by the swirling strength, revealing remarkable changes in the secondary flow structures. Strong secondary motions occurred above thin ridges when $(S-W) / \delta \approx \mathcal{O}(1)$, whose extent are shown to scale with the valley width, while their strength scale with the ridge curvature. For the rectangular ridges, a pair of new small counter-rotating vortices of opposed sign emerge above the ridges forming tertiary structures. For $(S-W) / \delta \leq 0.5$, an imbalance in favour of the tertiary vortices occurs which take over the secondary ones, leading to the swap of the LMPs and the HMPs locations. The results also indicated that the spanwise spacing alone is insufficient to characterise the surface heterogeneity. An additional parameter based on the ratio of perimeters of recessed and elevated regions, $\xi$, was adopted. It adequately captured the changes in shapes and widths of the ridges highlighting the effects of the heterogeneity on drag and the streamwise circulation in the outer-layer.

Triple decomposition of the velocity components allowed the examination of the surface heterogeneity through the dispersive stresses. We determined qualitatively and quantitatively the contributions of the form-induced stresses to the total shear-stress, which varied from $15 \%$ to $55 \%$, proportional to the strength of the secondary motions. The total shear-stress estimates were subsequently used to examine outer-layer similarity in the mean and turbulence statistics. The results indicated lack of similarity in the mean flow, however a collapse with the smooth-wall is observed in the diagnostic form. This observation indicates that the secondary motions affect proportionally the mean flow and the turbulent structure in the outer region.

The two-point correlation maps of the streamwise velocity fluctuations $R_{u u}$ allowed the examination of the turbulence structures in the outer region. The structures exhibit spanwise symmetry above the LMPs and HMPs, while, they are asymmetric in the wall-normal direction due to the intense inward/outward radial motions. In the middistance between the LMPs and HMPs, the turbulent structures exhibited a relatively higher coherence accompanied with a substantial inclination towards the HMPs, which is consistent with previous studies. The analysis of the TNTI revealed the impact of the secondary flows on the wake intermittency. Instantaneous snapshots showed the existence of strong bulges and valleys in the interface outline, which is translated in TNTI undergoing a spanwise modulation. The extent of the intermittency is shown to vary across the spanwise direction with a shorter and larger intermittency extents at the upwash and downwash regions, respectively. The global probability density function of the TNTI indicated that the main impact of the secondary flows is the change in the intermittency extent, with stronger secondary flows inducing a larger extent.

The authors acknowledge the financial support of the Engineering and Physical Sciences Research Council of the United Kingdom (EPSRC Grant Ref. No. EP/L006383/1 and EP/P021476/1). CV is supported by a Marie-Curie fellowship (project ref no. 656081). TM is also partially supported by the Faculty of Engineering and Physical Sciences at the University of Southampton. The authors would like to also acknowledge the insightful comments and pertinent observations of the referees during the review process of this manuscript. 


\section{REFERENCES}

Akomah, A., Hangan, H. \& Naughton, J. 2011 Very high reynolds number boundary layers over 3d sparse roughness and obstacles: the mean flow. Exp. Fluids 51 (3), 743.

Alfredsson, P. H., Segalini, A. \& Örlü, R. 2011 A new scaling for the streamwise turbulence intensity in wall-bounded turbulent flows and what it tells us about the "outer" peak. Phys. Fluids 23 (4), 041702.

Anderson, W., Barros, J. M., Christensen, K. T. \& Awasthi, A. 2015 Numerical and experimental study of mechanisms responsible for turbulent secondary flows in boundary layer flows over spanwise heterogeneous roughness. J. Fluid Mech 768, 316-347.

Anderson, W., Yang, J., Shrestha, K. \& Awasthi, A. 2018 Turbulent secondary flows in wall turbulence: vortex forcing, scaling arguments, and similarity solution. Environ. Fluid Mech 18 (6), 1351-1378.

Awasthi, A. \& Anderson, W. 2018 Numerical study of turbulent channel flow perturbed by spanwise topographic heterogeneity: Amplitude and frequency modulation within lowand high-momentum pathways. Phys. Rev. Fluids 3, 044602.

Bai, H. L., Kevin, K., Hutchins, N. \& Monty, J. P. 2018 Turbulence modifications in a turbulent boundary layer over a rough wall with spanwise-alternating roughness strips. Phys. Fluids 30 (5), 055105, arXiv: https://doi.org/10.1063/1.5026134.

Barros, J. M. \& Christensen, K. T. 2014 Observations of turbulent secondary flows in a rough-wall boundary layer. J. Fluid Mech $\mathbf{7 4 8}$.

Benschop, H. \& Breugem, W. 2017 Drag reduction by herringbone riblet texture in direct numerical simulations of turbulent channel flow. J. Turbul 18 (8), 717-759, arXiv: https://doi.org/10.1080/14685248.2017.1319951.

Bisset, D. K., Hunt, J. C. R. \& Rogers, M. M. 2002 The turbulent/non-turbulent interface bounding a far wake. J. Fluid Mech 451, 383-410.

Blay Esteban, L., Dogan, E., Rodríguez-Lopéz, E. \& Ganapathisubramani, B. 2017 Skin-friction measurements in a turbulent boundary layer under the influence of freestream turbulence. Exp. Fluids $\mathbf{5 8}$ (9), 115.

Castro, I. P. 2007 Rough-wall boundary layers: mean flow universality. J. Fluid Mech 585, 469-485.

Castro, I. P., Segalini, A. \& Alfredsson, P. H. 2013 Outer-layer turbulence intensities in smooth-and rough-wall boundary layers. J. Fluid Mech 727, 119-131.

Chan, L., MacDonald, M., Chung, D., Hutchins, N. \& Ooi, A. 2018 Secondary motion in turbulent pipe flow with three-dimensional roughness. J. Fluid Mech 854, 5-33.

Chauhan, K., Philip, J., De Silva, C. M., Hutchins, N. \& Marusic, I. 2014 The turbulent/non-turbulent interface and entrainment in a boundary layer. J. Fluid Mech 742, 119-151.

Chung, D., Monty, J. P. \& Hutchins, N. 2018 Similarity and structure of wall turbulence with lateral wall shear stress variations. J. Fluid Mech 847, 591-613.

Colombini, M. 1993 Turbulence-driven secondary flows and formation of sand ridges. J. Fluid Mech 254, 701-719.

Corrsin, T \& Kistler, A. L. 1955 Free-stream boundaries of turbulent flows. Naca technical report 1244. Washington, DC.

Djenidi, L., Talluru, K. M. \& Antonia, R. A. 2018 Can a turbulent boundary layer become independent of the reynolds number? J. Fluid Mech 851, 1-22.

Flack, K. A. \& Schultz, M. P. 2010 Review of hydraulic roughness scales in the fully rough regime. J. Fluids Engng 132 (4), 041203.

Flack, K. A. \& Schultz, M. P. 2014 Roughness effects on wall-bounded turbulent flowsa). Phys. Fluids 26 (10), 101305.

Goldstein, D. B. \& Tuan, T.-C. 1998 Secondary flow induced by riblets. J. Fluid Mech 363, $115-151$.

Gyr, A. \& Kinzelbach, W. 2004 Bed forms in turbulent channel flow. Appl. Mech. Rev 57 (1), $77-93$.

Hinze, J. O. 1967 Secondary currents in wall turbulence. Phys. Fluids 10 (9), 122-S12.

Hinze, J. O. 1973 Experimental investigation on secondary currents in the turbulent flow through a straight conduit. Appl. Sci. Res 28 (1), 453-465. 
Hwang, H. G. \& LEe, J. H. 2018 Secondary flows in turbulent boundary layers over longitudinal surface roughness. Phys. Rev. Fluids 3, 014608.

Jiménez, J. 2004 Turbulent flows over rough walls. Annu. Rev. Fluid Mech. 36, 173-196.

Kevin, K., Monty, J. \& Hutchins, N. 2019 Turbulent structures in a statistically threedimensional boundary layer. J. Fluid Mech 859, 543-565.

Kevin, K., Monty, J. P., Bai, H. L., Pathikonda, G., Nugroho, B., Barros, J. M., Christensen, K. T. \& Hutchins, N. 2017 Cross-stream stereoscopic particle image velocimetry of a modified turbulent boundary layer over directional surface pattern. $J$. Fluid Mech 813, 412-435.

Krug, D., Philip, J. \& Marusic, I. 2017 Revisiting the law of the wake in wall turbulence. J. Fluid Mech 811, 421-435.

Laskari, A., de Kat, R., Hearst, R. J. \& Ganapathisubramani, B. 2018 Time evolution of uniform momentum zones in a turbulent boundary layer. J. Fluid Mech 842, 554-590.

Mclelland, S. J., Ashworth, P. J., Best, J. L. \& Livesey, J. R. 1999 Turbulence and secondary flow over sediment stripes in weakly bimodal bed material. J. Hydraul. Engng 125 (5), 463-473.

Medjnoun, T., Vanderwel, C. \& Ganapathisubramani, B. 2018 Characteristics of turbulent boundary layers over smooth surfaces with spanwise heterogeneities. J. Fluid Mech 838, $516-543$.

Mejia-Alvarez, R. \& Christensen, K. T. 2013 Wall-parallel stereo particle-image velocimetry measurements in the roughness sublayer of turbulent flow overlying highly irregular roughness. Phys. Fluids 25 (11), 115109.

Meyers, J., Ganapathisubramani, B. \& Cal, R. B. 2019 On the decay of dispersive motions in the outer region of rough-wall boundary layers. J. Fluid Mech 862, R5.

Moinuddin, K. A. M., Joubert, P. N. \& Chong, M. S. 2004 Experimental investigation of turbulence-driven secondary motion over a streamwise external corner. J. Fluid Mech 511, 1-23.

Nagib, H. M., Chauhan, K. A. \& Monkewitz, P. A. 2007 Approach to an asymptotic state for zero pressure gradient turbulent boundary layers. Phil. Trans. R. Soc. Lond. A 365 (1852), 755-770.

Napoli, E., Armenio, V. \& De Marchis, M. 2008 The effect of the slope of irregularly distributed roughness elements on turbulent wall-bounded flows. J. Fluid Mech 613, 385394.

Naughton, J. W. \& Sheplak, M. 2002 Modern developments in shear-stress measurement. Prog. Aerosp. Sci 38 (6-7), 515-570.

Nezu, I. \& Nakagawa, H. 1984 Cellular secondary currents in straight conduit. J. Fluids Engng 110 (2), 173-193.

Nezu, I., Tominaga, A. \& Nakagawa, H. 1993 Field measurements of secondary currents in straight rivers. J. Fluids Engng 119 (5), 598-614.

Nikora, V., McEwan, I., Mclean, S., Coleman, S., Pokrajac, D. \& Walters, R. 2007 Double-averaging concept for rough-bed open-channel and overland flows: Theoretical background. J. Hydraul. Engng 133 (8), 873-883.

NikURADSE, J. 1930 Untersuchungen über turbulente strömungen in nicht kreisförmigen rohren. Ing-Arch 1 (3), 306-332.

Nugroho, B., Hutchins, N. \& Monty, J. P. 2013 Large-scale spanwise periodicity in a turbulent boundary layer induced by highly ordered and directional surface roughness. Intl J. Heat Fluid Flow 41, 90-102.

Pailhas, G., Barricau, P., Touvet, Y. \& Perret, L. 2009 Friction measurement in zero and adverse pressure gradient boundary layer using oil droplet interferometric method. Exp. Fluids 47 (2), 195-207.

Pathikonda, G. \& Christensen, K. T. 2017 Inner ${ }^{\circ}$ outer interactions in a turbulent boundary layer overlying complex roughness. Phys. Rev. Fluids 2, 044603.

Placidi, M. \& Ganapathisubramani, B. 2015 Effects of frontal and plan solidities on aerodynamic parameters and the roughness sublayer in turbulent boundary layers. $J$. Fluid Mech 782, 541-566.

Prandth, L. 1952 Essentials of fluid dynamics. Blackie. 
RAUPACH, M. R. \& Shaw, R. H. 1982 Averaging procedures for flow within vegetation canopies. Boundary-Layer Met 22 (1), 79-90.

Reynolds, R. T., Hayden, P., Castro, I. P. \& Robins, A. G. 2007 Spanwise variations in nominally two-dimensional rough-wall boundary layers. Exp. Fluids 42 (2), 311-320.

Reynolds, W. C. \& Hussain, A. K. M. F. 1972 The mechanics of an organized wave in turbulent shear flow. part 3. theoretical models and comparisons with experiments. $J$. Fluid Mech 54 (2), 263-288.

Schlichting, H. 1979 Boundary-layer theory, 7th edn. McGraw-Hill.

Shockling, M. A., Allen, J. J. \& Smits, A. J. 2006 Roughness effects in turbulent pipe flow. J. Fluid Mech 564, 267-285.

Squire, D. T., Morrill-Winter, C., Hutchins, N., Schultz, M. P., Klewicki, J. C. \& Marusic, I. 2016 Comparison of turbulent boundary layers over smooth and rough surfaces up to high reynolds numbers. J. Fluid Mech 795, 210-240.

Squire, L. C. 1961 The motion of a thin oil sheet under the steady boundary layer on a body. J. Fluid Mech 11, 161-179.

Stroh, A., Hasegawa, Y., Kriegseis, J. \& Frohnapfel, B. 2016 Secondary vortices over surfaces with spanwise varying drag. J. Turbul 17 (12), 1142-1158.

TAnner, L. H. \& Blows, L. G. 1976 A study of the motion of oil films on surfaces in air flow, with application to the measurement of skin friction. J. Phys E 9 (3), 194.

Thakkar, M., Busse, A. \& Sandham, N. 2017 Surface correlations of hydrodynamic drag for transitionally rough engineering surfaces. J. Turbul 18 (2), 138-169, arXiv: https://doi.org/10.1080/14685248.2016.1258119.

Townsend, A. A. 1976 The structure of turbulent shear flow, 2nd edn. Cambridge University Press.

Türk, S., Daschiel, G., Stroh, A., Hasegawa, Y. \& Frohnapfel, B. 2014 Turbulent flow over superhydrophobic surfaces with streamwise grooves. J. Fluid Mech 747, 186-217.

Vanderwel, C. \& Ganapathisubramani, B. 2015 Effects of spanwise spacing on large-scale secondary flows in rough-wall turbulent boundary layers. J. Fluid Mech 774, R2.

Vanderwel, C., Placidi, M. \& Ganapathisubramani, B. 2017 Wind resource assessment in heterogeneous terrain. Phil. Trans. R. Soc. Lond. A 375 (2091), 20160109.

Vanderwel, C., Stroh, A., Kriegseis, J., Frohnapfel, B. \& Ganapathisubramani, B. 2019 The instantaneous structure of secondary flows in turbulent boundary layers. J. Fluid Mech 862, 845-870.

Wang, Z. Q. \& Cheng, N. S. 2005 Secondary flows over artificial bed strips. Adv. Water Resour 28 (5), 441-450.

WANG, Z. Q. \& Cheng, N. S. 2006 Time-mean structure of secondary flows in open channel with longitudinal bedforms. Adv. Water Resour 29 (11), 1634-1649.

Westerweel, J., Fukushima, C., Pedersen, J. M. \& Hunt, J. C. R. 2005 Mechanics of the turbulent-nonturbulent interface of a jet. Phys. Rev. Lett. 95, 174501.

Willingham, D., Anderson, W., Christensen, K. T. \& Barros, J. M. 2014 Turbulent boundary layer flow over transverse aerodynamic roughness transitions: induced mixing and flow characterization. Phys. Fluids 26 (2), 025111.

Wu, Y. \& Christensen, K. T. 2007 Outer-layer similarity in the presence of a practical rough-wall topography. Phys. Fluids 19 (8), 085108.

Wu, Y. \& Christensen, K. T. 2010 Spatial structure of a turbulent boundary layer with irregular surface roughness. J. Fluid Mech 655, 380-418.

YANG, J \& ANDERSON, W 2017 Numerical study of turbulent channel flow over surfaces with variable spanwise heterogeneities: Topographically-driven secondary flows affect outerlayer similarity of turbulent length scales. Flow Turbul. Combust.

YuAn, J. \& Piomelli, U. 2014 Roughness effects on the reynolds stress budgets in near-wall turbulence. J. Fluid Mech 760, R1. 